\title{
Pressure-Volume-Temperature Relationships for Normal Deuterium Between 18.7 and 21.0 K
}

\author{
L. A. Schwalbe and E. R. Grilly \\ Los Alamos National Laboratory, Los Alamos, NM 87545
}

\begin{abstract}
Accepted: December 19. 1983
Analytical expressions are derived for the melting line and liquid equation of state of normal deuterium near the triple point. Melting pressures were measured between the triple point and $20.4 \mathrm{~K}$. These results combined with existing pressure measurements along the saturated liquid-vapor curve fix an accurate value, $T_{\mathrm{tp}}=18.723 \mathrm{~K}$, for the triple-point temperature. Data for the isothermal compressibility and thermal expansion coefficients of the liquid were taken over the temperature and pressure ranges 18.8 to $21.0 \mathrm{~K}$ and 4 to $70 \mathrm{bar}$, respectively. The liquid molar volume was measured at nine points below $20.4 \mathrm{~K}$. All liquid PVT data are shown to be internally consistent. Measurements of the volume changes on melting are also presented. The heat of fusion and the solid molar volume at melting are deduced from these data. Also included are detailed comparisons of our results with existing data. A critical appraisal is given of all measured thermodynamic quantities in this regime.
\end{abstract}

Key words: compressibility; deuterium; equation of state; melting pressure; ortho-para concentration; pressurevolume-temperature (PVT); thermal expansion; thermodynamic properties; triple point.

\section{Introduction}

Interest in the properties of the condensed-phase hydrogen systems has continued for well over 50 years. As early as 1935, Clusius and Bartholome [1]' published the first comprehensive thermodynamic study of normal deuterium $\left(n-D_{2}\right)$ in the triple-point region. These results were followed by others [2,3] and included in the 1948 review article by Woolley et al.[4]. Since that time, there have been some additional measurements in this regime $[5,6]$, but most efforts have been toward establishing the pressure-volume-temperature (PVT) relationship over extended ranges of temperatures and pressures.

By 1959 , an incomplete collection of fluid-phase density data was available at various intervals in the

About the Authors, Paper: The work reported on was performed at the Los Alamos National Laboratory where L. A. Schwalbe, a physicist, remains and from which E. R. Grilly, also a physicist, is retired. The work was supported by the Fusion Target Fabrication Group at Los Alamos.

\footnotetext{
${ }^{1}$ Figures in brackets indicate literature references at the end of this paper.
}

temperature range from the triple point to $420 \mathrm{~K}$ and at pressures to $3 \mathrm{kbar}$. Prydz [7,8] critically reviewed these results, fit them to a modified Strobridge equation, and derived analytical expressions for selected thermodynamic properties.

More recently Mills et al. [9] measured simultaneously the molar volume and ultrasound velocity of fluid $n-D_{2}$ in a piston-cylinder apparatus. The data spanned temperatures of 75 to $300 \mathrm{~K}$ and pressures of 2 to $20 \mathrm{kbar}$. The results were fit to a Benedict-type equation of state, and analytic forms were derived for both constant-volume and constant-pressure heat capacities over these ranges. In a companion to this work, Liebenberg et al. [10] reported measurements of the melting line from 4 to $19 \mathrm{kbar}$ and the corresponding changes in the molar volume and longitudinal sound velocity. From these data, they derived the entropy change on melting, the adiabatic compressibility for the solid and other thermodynamic properties.

Most work on solid deuterium has been done at low temperatures and low pressures although the volumepressure dependence at $4 \mathrm{~K}$ was measured to 20 [11] and $25 \mathrm{kbar}$ [12]. Recently, solid-phase isochoric equationof-state data were taken to $2 \mathrm{kbar}[13,14]$. 
The purpose of this study is to supplement existing PVT and melting data with accurate and precise measurements ${ }^{2}$ in the region of the phase diagram near the triple point. Data are presented for the pressures and liquid molar volume of $n-D_{2}$ along the melting line from the triple point to $20.4 \mathrm{~K}$. The isothermal compressibility and thermal expansion coefficients of the liquid are given over the same temperature range and at pressures between 4 and 70 bar. The combined liquid data are fitted to an empirical equation of state and are shown to be internally consistent.

Measurements of the volume changes on melting are also presented. The heat of fusion and the solid molar volume at melting are deduced from these data. Throughout the discussion, we have included detailed comparisons of our results with existing data and have attempted to provide a critical appraisal of all measured quantities in this regime.

\section{Experimental}

Our studies on $n-D_{2}$ were carried out with essentially the same apparatus as that used earlier for similar work [15-17] on ${ }^{3} \mathrm{He}$ and ${ }^{4} \mathrm{He}$. The pressure cell, which was referred to in previous descriptions [16,17] as "cell I," consisted of three $\mathrm{BeCu}$ diaphragms that were welded together at their circumference and separated by $0.3 \mathrm{~mm}$ gaps (see fig. 1). The lower gap was connected to a capillary tube and remained open to a room-temperature helium gas-handling system. With this arrangement $P_{1}$, the pressure of the lower-cell volume, could be adjusted or measured directly. The upper cell gap served as the deuterium sample chamber; a fixed quantity of material could be maintained in its volume $V_{\mathrm{u}}$ by sealing the low-temperature valve. The sample pressure $P_{\mathrm{u}}$ was determined from the deflection of the upper diaphragm. Originally the deflection measurement was made with a linear differential transformer [15], but the capacitance technique was adopted because of its greater sensitivity [17].

Changes in the volume of the sample chamber depend upon changes in $P_{\mathrm{u}}, P_{\mathrm{l}}$, and the bath pressure $P_{\mathrm{b}}$ according to the equation

$$
\Delta V_{\mathrm{u}}=\left(S_{\mathrm{u}}+S_{\mathrm{l}}\right) \Delta P_{\mathrm{u}}-S_{1} \Delta P_{1}-S_{\mathrm{u}} \Delta P_{\mathrm{b}}
$$

where $S_{\mathrm{u}}$ and $S_{1}$ are pressure sensitivity factors of the volume changes to changes in the upper and middle diaphragm displacements, respectively.

The pressure sensitivity factors for the cell were not constants but varied reproducibly by 1 or $2 \%$ as $P_{1}$ and $P_{\mathrm{u}}$ varied between 0 and 70 bars, the highest working pressures in this experiment. For the earlier helium measurements, these variations did not critically affect the results, but for the present work it was first necessary to calibrate the system more precisely. To do this, three separate sets of calibration measurements were run. In each, the procedure first involved loading the sample chamber with liquid $\mathrm{p}-\mathrm{H}_{2}$ at $T=20.00 \mathrm{~K}$ and then "bleeding" small amounts from the cell through the valve and into a calibrated standard volume held nominally at room temperature. The quantity of material drawn from the cell was then measured and the corresponding changes in the sample volume $\Delta V_{\mathrm{u}}$ were calculated using the $\mathrm{p}-\mathrm{H}_{2}$ density values tabulated by Goodwin et al. [18]. According to eq (1), isothermal changes

${ }^{2}$ Full data are available from the authors.

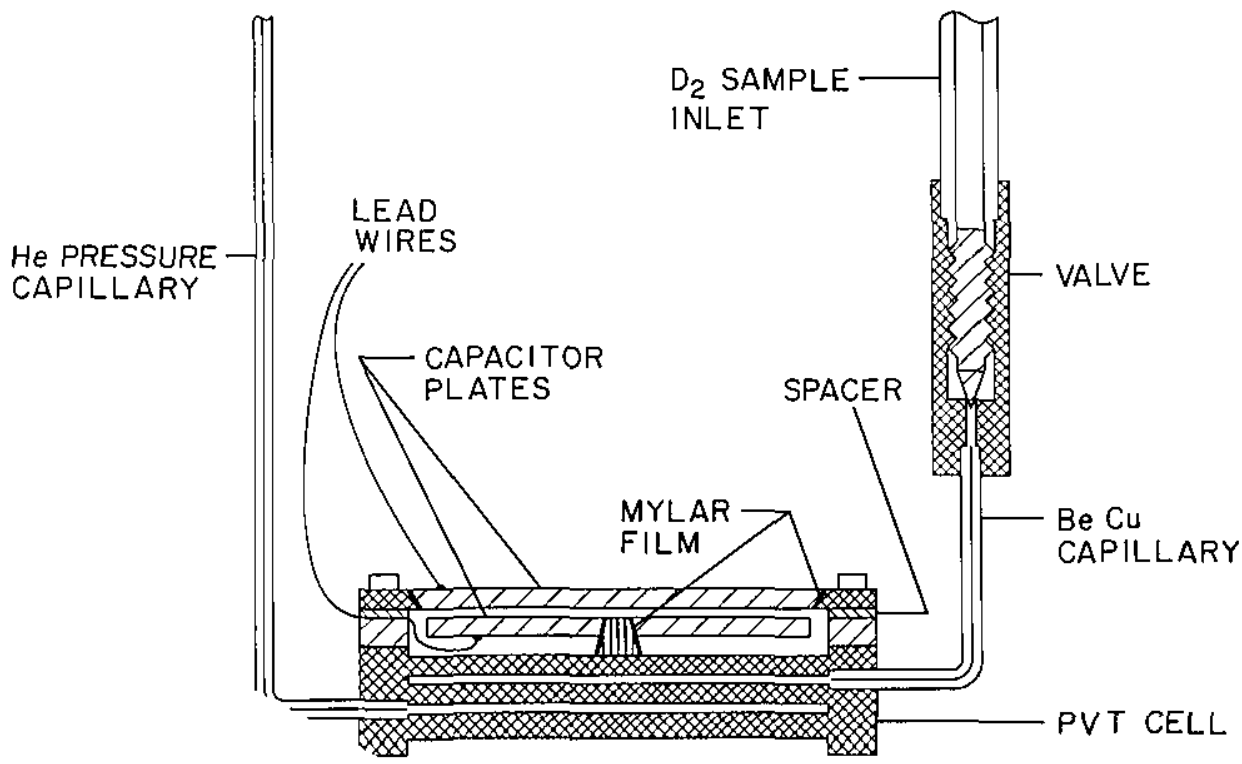

Figure 1-The pressure cell and low-temperature valve assembly. 
in $V_{\mathrm{u}}$ under the constraints $\Delta P_{\mathrm{u}}=0, \Delta P_{\mathrm{u}}=\Delta P_{1}$, and $\Delta P_{1}=0$ yield the factors $S_{\mathrm{l}}, S_{\mathrm{u}}$, and $S_{\mathrm{u}}+S_{\mathrm{l}}$, respectively.

A consistent set of pressure sensitivity parameters was obtained at $T=20.000 \mathrm{~K}$ for the entire working range of both upper- and lower-cell pressures. The quantities $S_{\mathrm{u}}$ and $S_{1}$ that were used in the data analysis were accurate to $\pm 0.1 \%$. Extrapolations of total volume measurements to $P_{\mathrm{u}}=P_{1}=P_{\mathrm{b}}=0$ absolute pressure determined $V_{\mathrm{u} 0}$, the zero-pressure volume of the sample chamber. The latter calibration was made at two separate times: initially, before any of the deuterium work was begun and again near its completion about 18 months later. The first set of measurements obtained an average value $0.37477 \mathrm{~cm}^{3}$ for six separate runs; for the second set the average of five runs was $0.37426 \mathrm{~cm}^{3}$. In the data analysis we used the mean of these two values, $V_{0}=0.37452 \mathrm{~cm}^{3}$, and set an accuracy limit of $\pm 0.15 \%$ for this quantity. This error includes the $\pm 0.1 \%$ uncertainty of the original p- $\mathrm{H}_{2}$ density data [18].

Equation (1) has no explicit temperature dependence included. In this range, there are negligible effects introduced by the temperature coefficient of the cell material. The thermal expansion of $\mathrm{BeCu}$ should be comparable to that of $\mathrm{Cu}$. Rubin et al. [19] reported a linear expansion coefficient of $6 \times 10^{-6} \mathrm{~K}^{-1}$ for copper at $25 \mathrm{~K}$. The effects introduced by thermal expansion are, therefore, about an order of magnitude smaller than the quoted uncertainties in our data. To check this explicitly, we measured thermal expansion coefficients of liquid hydrogen in our cell. Hydrogen data were taken at $0.4 \mathrm{~K}$ intervals between $19.0 \mathrm{~K}$ and $21.0 \mathrm{~K}$ along six isobars $(5.48,14.76,28.52,42.28,56.05$, and 69.81 bar). The measured values agree with those that Roder et al. [20] derived from the original density data of Goodwin et al. [18].

The uniform sample temperatures required for this study were maintained by holding the pressure cell assembly immersed in a bath of liquid $20 \mathrm{~K}$-equilibrium hydrogen $\left(\mathrm{e}-\mathrm{H}_{2}\right)$. Temperatures were controlled by regulating the bath pressure with a Baratron ${ }^{3}$ Type 77H-1000 metal diaphragm gauge (0-1000 Torr range). Bath pressures were obtained directly with an 18-mm-bore mercury manometer. The mercury column heights were measured with a cathetometer, and standard corrections for the temperature and local gravity constant were applied to these readings. From these data the sample temperatures were calculated with the

\footnotetext{
${ }^{3}$ Certain trade names and company products are identified in order to adequately specify the experimental procedure. In no case does such identification imply recommendation or endorsement by the Los Alamos National Laboratory or the Journal of Research of the National Bureau of Standards, nor does it imply that the product is necessarily the best available for the purpose.
}

saturated liquid-vapor pressure equation of Souers et al. [21]:

$$
\begin{gathered}
\ln P_{\mathrm{b}}(\text { torr })=10.57411-101.3378 T^{-1} \\
+5.432005 \times 10^{-2} T-1.105632 \times 10^{-4} T^{2} .
\end{gathered}
$$

This function is identical to that defined by the International Practical Temperature Scale of 1968 (IPTS-68) [22] although it is expressed in somewhat different form.

Temperature precisions of slightly better than $1 \mathrm{mK}$ were attainable with this procedure. From run to run, with the possible exceptions noted below, the scale seems to have been reproducible to within about \pm 2 $\mathrm{mK}$.

\section{Results and Discussion}

\subsection{Melting Pressures}

The melting pressures of $n-D_{2}$ were measured by closing the cell valve on a liquid-phase sample and increasing $P_{1}$ until the capacitance readings showed the sample to be in the two-phase region. At each temperature, capacitance readings were recorded for several different $P_{1}$ values. Finally, the valve was opened and the capacitance-pressure calibration was rechecked against the piston gauge. The results are given in table 1 . Measurements were taken during four runs, each lasting one week; each run represents a separate filling of e- $\mathrm{H}_{2}$ refrigerant.

It is important to note that all melting pressures reported here correspond to the "first-freeze" characteristics of the sample. The pressure-temperature measurements were recorded for small solid fractions $(<5 \%)$ of the two-phase mixture. In a later section, we discuss in more detail the problems involved with precision measurements of the sample pressure when larger relative quantities of solid are present.

Values for the slope of the melting curve are plotted in figure 2. Those obtained from run numbers $1,2,3 \mathrm{~b}$, and 4 were derived from consecutive melting pressure measurements. Those labeled " $3 a$ " were measured directly by temperature cycling a single sealed sample. The plot indicates a nearly linear temperature dependence for the slope. The melting line $P_{\mathrm{m}}\left(T_{\mathrm{m}}\right)$ should, therefore, be adequately represented by a quadratic function over this limited range. For the least-squares fit, we chose an equation of the form

$$
P_{\mathrm{m}}\left(T_{\mathrm{m}}\right)=P_{\mathrm{tp}}+A_{1}\left(T_{\mathrm{m}}-T_{\mathrm{tp}}\right)+A_{2}\left(T_{\mathrm{m}}-T_{\mathrm{tp}}\right)^{2}
$$

where $A_{1}$ and $A_{2}$ are fitting constants and $P_{\mathrm{tp}}$ and $T_{\mathrm{tp}}$ are parameters that represent the pressure and temperature values at the triple point. Our best fit gave $A_{1}=38.884$ 
Table 1. Measured pressure and temperatures of normal deuterium along the melting line.

\begin{tabular}{|c|c|c|c|c|c|c|c|c|c|c|c|}
\hline Run No. 1 & $T_{\mathrm{m}}(\mathrm{K})$ & $P_{\mathrm{m}}(\mathrm{bar})$ & Run No. 2 & $T_{\mathrm{m}}(\mathrm{K})$ & $P_{\mathrm{m}}$ (bar) & Run No. 3 & $T_{\mathrm{m}}(\mathrm{K})$ & $P_{\mathrm{m}}$ (bar) & Run No. 4 & $T_{\mathrm{m}}(\mathrm{K})$ & $P_{\mathrm{m}}$ (bar) \\
\hline & $20.407_{7}$ & 69.61 & & $19.504_{2}$ & 31.90 & & $19.703_{0}$ & 40.03 & & $20.306_{4}$ & 65.16 \\
\hline & $20.206_{0}$ & 60.75 & & $19.302_{4}$ & 23.73 & & $20.407_{0}$ & 69.44 & & $20.103_{1}$ & 56.62 \\
\hline & $20.006_{6}$ & 52.40 & & $19.102_{2}$ & 15.72 & & $20.204_{3}$ & 60.86 & & $19.902_{4}$ & 48.24 \\
\hline & $19.805_{7}$ & 44.02 & & $18.902_{0}$ & 7.81 & & 20.004 , & 52.48 & & $19.699_{s}$ & 39.86 \\
\hline & 19.605 & 36.10 & & $20.007_{0}$ & 52.57 & & $19.802_{2}$ & 44.08 & & $19.504_{4}$ & 31.88 \\
\hline & $19.404_{6}$ & 27.88 & & $19.804_{2}$ & 44.14 & & 19.1927 & 19.40 & & $19.301_{9}$ & 23.68 \\
\hline & $19.198_{2}$ & 19.61 & & $19.603_{8}$ & 35.93 & & $19.000_{9}$ & 11.75 & & $19.100_{4}$ & 15.64 \\
\hline & 20.3069 & 65.29 & & 19.4029 & 27.75 & & & & & $18.899_{2}$ & 7.69 \\
\hline & $20.107_{1}$ & 56.83 & & $20.407_{6}$ & 69.44 & & & & & 19.404 & 27.79 \\
\hline & $19.906_{0}$ & 48.12 & & $20.305_{6}$ & 65.10 & & & & & 19.195: & 19.38 \\
\hline & $19.704_{6}$ & 39.79 & & $20.205_{6}$ & 60.83 & & & & & 19.000 & 11.65 \\
\hline & \multirow[t]{3}{*}{$20.408_{1}$} & 69.87 & & $20.105_{4}$ & 56.63 & & & & & $18.829_{2}$ & 4.88 \\
\hline & & & & $19.906_{2}$ & 48.05 & & & & & $19.603_{8}$ & 36.04 \\
\hline & & & & & & & & & & $18.828_{7}$ & 4.96 \\
\hline
\end{tabular}

$\mathrm{bar} / \mathrm{K}, A_{2}=1.078 \mathrm{bar} / \mathrm{K}^{2}$, and $T_{\mathrm{tp}}=18.7067 \mathrm{~K}$ with $P_{\mathrm{tp}}=0.17$ bar.

Grilly [23] reported a triple-point temperature of $18.73 \mathrm{~K}$. The result obtained here $18.7067 \mathrm{~K}$ is considerably lower. We attempted to fit eq (3) by varying only $A_{1}$ and $A_{2}$ with $T_{\mathrm{tp}}=18.73 \mathrm{~K}$ and $P_{\mathrm{tp}}=0.17$ bar held fixed, but the results showed large systematic deviations. We

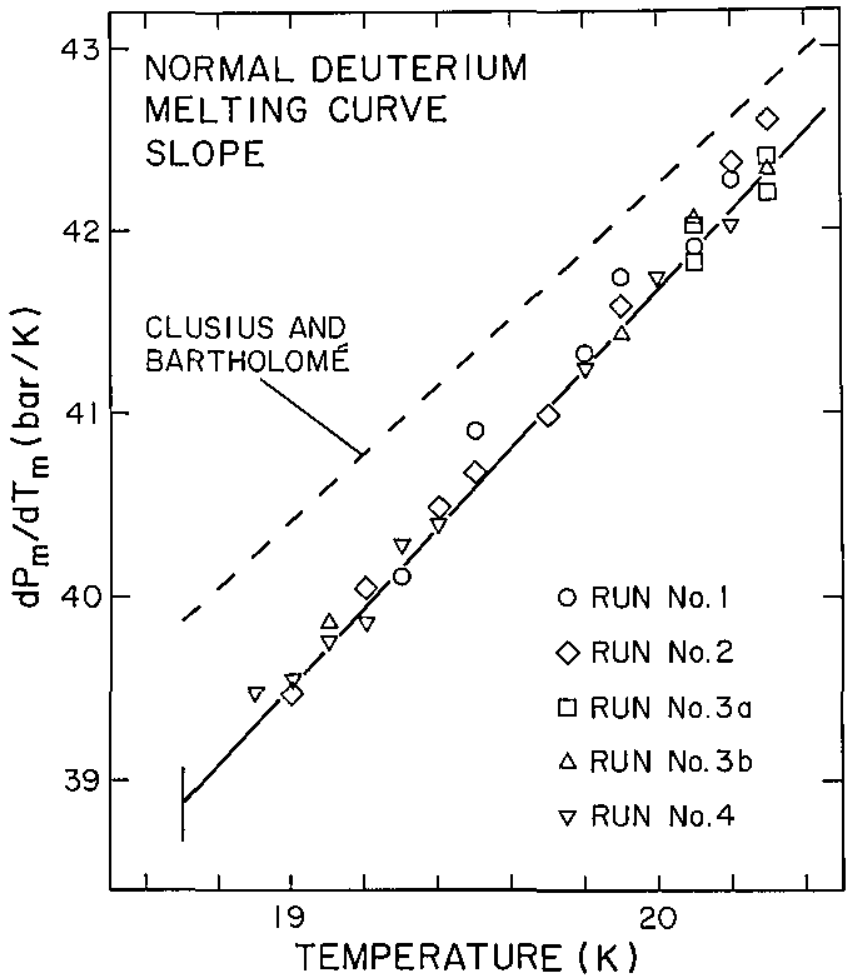

Figure 2-The slope of the melting curve. Data from runs 1, 2, 3b, and 4 were obtained by subtracting consecutive measurements of the melting pressure. Data from run $3 a$ were measured by temperature cycling a single sealed sample. The solid line was obtained by evaluating eq (4). The dashed line is the slope of the melting function given by Clusius and Bartholome [1]. believe the difference is due to impurity effects in the actual fixed-point values of our sample lot. Mass spectrometric analysis of the sample material showed $0.75 \%$ HD present with no detectable traces of $\mathrm{H}_{2}, \mathrm{~N}_{2}$, or $\mathrm{H}_{2} \mathrm{O}$ at the $50 \mathrm{ppm}$ sensitivity level. Sample impurities in low concentrations affect both triple-point parameters, but within the resolution limits of this experiment, the temperature $T_{\mathrm{tp}}$ is much more sensitively dependent on impurity effects than is the pressure $P_{\mathrm{tp}}$. Therefore, in the final calculation we retained the fixed value $P_{\mathrm{tp}}=0.17$ bar and allowed only $T_{\text {tp }}$ to vary along with $A_{1}$ and $A_{2}$.

Equation (3) with the above parameters describes the melting line for our sample, but for pure n- $D_{2}$, a pressure correction must be applied. If this correction is proportional to the HD concentration and to the difference in pressure between the $\mathrm{HD}$ and $\mathrm{D}_{2}$ melting curves, $0.6 \mathrm{bar}$ should be subtracted from $P_{\mathrm{m}}$ in eq (3) to obtain the melting pressure of pure $\mathrm{n}-\mathrm{D}_{2}$.

A plot of the deviations of our data from eq (3) is shown in figure 3. With the exception of a few points, mainly all from the first run, the data appear to lie within \pm 0.1 bar of the smooth curve. The precision of our measurements is comparable to that of Bereznyak and Sheinina [24], who measured the melting lines of e- $\mathrm{H}_{2}$, $\mathrm{n}-\mathrm{H}_{2}$, and e- $\mathrm{D}_{2}$ over roughly the same pressure interval. Their $P_{\mathrm{m}}$ measurements on e- $\mathrm{D}_{2}$ exceed ours on $\mathrm{n}-\mathrm{D}_{2}$ by 1.5 bar at $18.8 \mathrm{~K}$ and 2.3 bar at $21.0 \mathrm{~K}$. (This difference is similar to that observed between the melting lines of e- $\mathrm{H}_{2}$ and $\mathrm{n}-\mathrm{H}_{2}$.) However, they observed a slope discontinuity in their e- $\mathrm{D}_{2}$ melting curve at $T=19.02 \mathrm{~K}$ and $P=14.2$ bar. Our data show no anomalous behavior anywhere in the range 18.7067 to $20.400 \mathrm{~K}$.

An analytic form for the slope of the melting curve is obtained by differentiating eq (3) with respect to $T_{\mathrm{m}}$ :

$$
\frac{\mathrm{d} P_{\mathrm{m}}}{\mathrm{d} T_{\mathrm{m}}}=A_{1}+2 A_{2}\left(T_{\mathrm{m}}-T_{\mathrm{tp}}\right)
$$




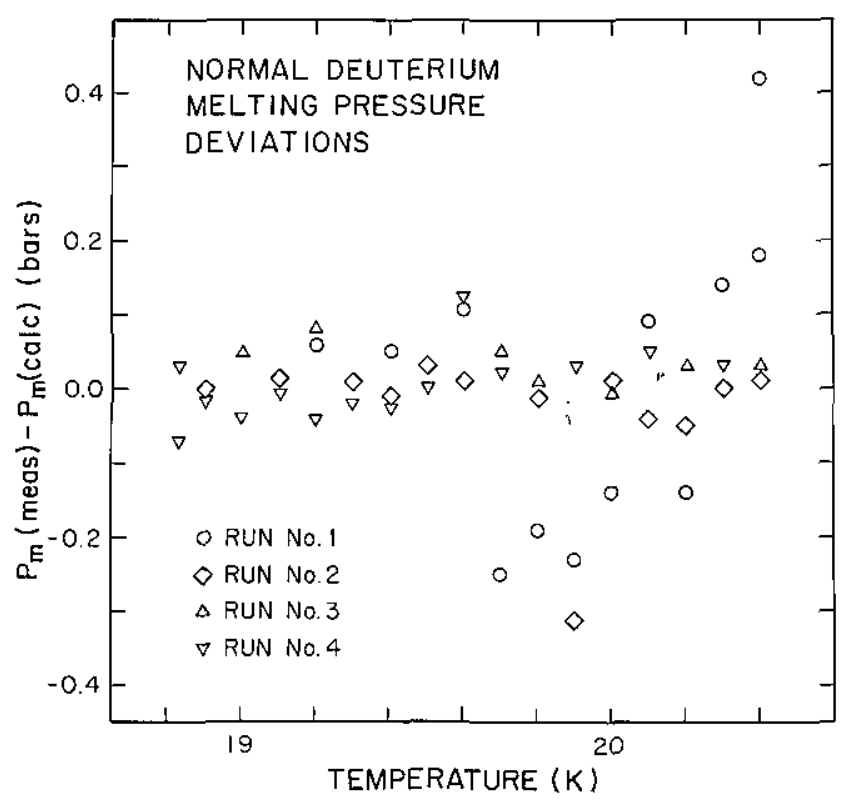

Figure 3-The deviations of the melting pressure data from the smooth curve defined by eq (3).

We have included in figure 2 a plot of eq (4) with the values $A_{1}, A_{2}$, and $T_{\text {tp }}$ found above. This function represents the data for the slope of the melting curve to within $\pm 0.5 \%$.

Other melting data are available for comparison. Among the earliest of these are the Clusius and Bartholome [1] results on $\mathrm{n}-\mathrm{D}_{2}$. These measurements extend from the triple point up in pressure to about 100 bar. Clusius and Bartholome employed a technique in which the liquid sample was held at nominally constant pressures while the surrounding $\mathbf{n}-\mathrm{H}_{2}$ bath pressure was gradually lowered. The freezing transition temperature was measured by observing the effect of the latent heat of the sample on the cryogen bath pressure. Their quoted temperatures were obtained from the measured cryogen vapor pressure and the centigrade-scale, $\theta(P)$, vapor-pressure equation of Keesom et al: [25]. Absolute temperatures, according to Bartholome [2], were then calculated from the expression $T(\mathrm{~K})=\theta+273.15$. Clusius and Bartholome fit their results to a Simon-type melting equation. This curve, corrected to the IPTS-68, is seen to lie about 2 bar higher than the present measurements.

Later studies have considerably extended the pressure range for the deuterium melting line. Chester and Dugdale [26] measured $\mathrm{n}-\mathrm{H}_{2}$ and $\mathrm{n}-\mathrm{D}_{2}$ to $2.8 \mathrm{kbar}$, but their results cannot be used for direct comparison here because the data were expressed only as melting pressure differences between the two isotopes at the same temperature. They observed a nearly constant difference of $167 \pm 8$ bar between 25 and $57 \mathrm{~K}$.
Further results were reported by Mills and Grilly $[27,28]$ who used a blocked-capillary technique. Their first data set [27], on $n-H_{2}$ and $n-D_{2}$ only, was limited in pressure to $1.5 \mathrm{kbar}$ because of embrittlement and cracking failures in the measurement apparatus. However, a later, improved system [28] allowed them to measure pressures of $n-H_{2}, n-D_{2}$, and $n-T_{2}$ to $3.5 \mathrm{kbar}$. Their deuterium samples had about the same HD concentration as ours. Below 70 bar, the limit of the present study, they obtained three points which we corrected to IPTS- 68 by adding $6 \mathrm{mK}$ to their temperatures. Comparison of these results with eq (3) shows their $P_{\mathrm{m}}$ measurements falling lower than our smooth curve by 0.4 to 0.8 bar. A comparison of the melting curves obtained for $\mathrm{n}-\mathrm{H}_{2}$ and $\mathrm{n}-\mathrm{D}_{2}$ shows a difference of about 170 bar near $20 \mathrm{~K}$.

Goodwin [29] proposed the empirical function

$$
\left(P_{\mathrm{m}}-P_{\mathrm{tp}}\right) /\left(T_{\mathrm{m}}-T_{\mathrm{tp}}\right)=A \exp \left(-a / T_{\mathrm{m}}\right)+B T_{\mathrm{m}}+C
$$

as a universal equation for the melting lines of the three hydrogen isotopes. For $C=0$, the constants $A, a$, and $B$ were fixed from existing normal and para-hydrogen melting data. The equation was then applied to $n-D_{2}$ and $\mathrm{n}-\mathrm{T}_{2}$ using their respective triple-point parameters, $T_{\mathrm{tp}}$ and $P_{\mathrm{tp}}$. Goodwin adjusted the values of $C$ to obtain best fits to the melting data of the two heavy isotopes. At pressures exceeding 100 bar, the function for deuterium was made to agree with the melting equation published by Mills and Grilly [28] to better than $0.5 \%$. Goodwin's equations for $n-\mathrm{H}_{2}$ and $n-\mathrm{D}_{2}$ differ in pressure by 169 bar in the $20 \mathrm{~K}$ region.

Several years after this work was done, Younglove [30] obtained additional melting data on $\mathrm{p}-\mathrm{H}_{2}$ and found these to be systematically offset from Goodwin's original function [29,31]. At low pressures, Younglove's melting pressures are about 0.4 bar lower than Goodwin's, and at high pressures (ca. 130 bar) they are 1.2 bar lower. It is, therefore, interesting to compare our measurements on $n-D_{2}$ with eq (5). In doing so, we find the temperature-corrected [32] form of Goodwin's equation to be about 0.5 to 0.8 bar lower than eq (3). However, the earlier function refers to a triple-point temperature of $18.72 \mathrm{~K}$, which more nearly represents the pure $n-D_{2}$ value than does our direct measurement of $18.7067 \mathrm{~K}$. The impurity correction of -0.6 bar discussed above brings the two melting curves into nearly complete correspondence. We suggest that Goodwin's equation describes the melting pressures of pure $n-D_{2}$ to about \pm 0.2 bar at pressures below 70 bar.

The only other melting curve available is that of Liebenberg et al. [10]. Although the range of validity of this experimental curve extends to $20 \mathrm{kbar}$, there are large 
( $\approx 40$ bar) discrepancies in the low-pressure range of present interest.

\subsection{Triple-point Temperature}

As we have mentioned, the physical significance of $T_{\text {tp }}$ in eq (3) is that it represents an empirical triple-point temperature for our sample material. A review and discussion of existing triple-point data for deuterium is presented separately [33].

Most of the earlier triple-point measurements were derived from saturated solid- and liquid-vapor pressure data. With this approach, separate empirical functions are fitted to the data in both solid and liquid temperature regimes. Simultaneous solution of these functions then defines the two triple-point parameters. In general, this technique allows $P_{\mathrm{tp}}$ to be fixed with precisions comparable to those of the direct pressure measurements. However, the temperature value at the intersection point is difficult to find closer than about 20 or $30 \mathrm{mK}$ [33]. The greater relative uncertainty in $T_{\mathrm{tp}}$ results from the small difference in slope between the solid and liquid vapor pressure functions.

Greater precision for $T_{\mathrm{p}}$ can be obtained with supplementary meiting data because the slope of the melting curve is very much larger than that of either the solid or liquid vapor pressure functions. Even with a relatively approximate estimate of the triple-point pressure, the temperature $T_{\mathrm{tp}}$ should be determined quite precisely. (For example, an error of $25 \%$ in $P_{\mathrm{tp}}$ would offset $T_{\mathrm{tp}}$ by only about $0.001 \mathrm{~K}$.) By similar argument it is clear that imprecise estimates of $T_{\mathrm{tp}}$ taken as fixed parameters in eq (3) should introduce systematic deviations in the residual plot corresponding to figure 3 . We examined this effect explicitly and found that significant deviations are apparent in the fitting results for all fixed $T_{t p}$ values that lie outside the range $18.7067 \pm 0.002 \mathrm{~K}$.

This value cannot be taken as the triple-point temperature of pure $n-D_{2}$ because the $H D$ impurity introduces some offset. Bereznyak et al. [34] published melting diagrams for mixtures of $\mathrm{p}-\mathrm{H}_{2} / \mathrm{o}-\mathrm{D}_{2}, \mathrm{p}-\mathrm{H}_{2} / \mathrm{HD}$, and $\mathrm{o}-\mathrm{D}_{2} / \mathrm{HD}$. The melting-temperature function of isotopic concentration for the $\mathrm{o}-\mathrm{D}_{2} / \mathrm{HD}$ system was found to be nearly linear between the triple points of the two pure elements. It is, therefore, reasonable to assume that a small concentration of HD-impurity $c(\mathrm{HD})$ in otherwise pure $n-D_{2}$ will shift the observed triple-point temperature from the pure-component value by an amount

$$
\Delta T_{\mathrm{tp}}=c(\mathrm{HD})\left[T_{\mathrm{tp}}(\mathrm{HD})-T_{\mathrm{tp}}\left(\mathrm{n}-\mathrm{D}_{2}\right)\right]
$$

Substituting $T_{\mathrm{tp}}(\mathrm{HD})=16.60 \mathrm{~K}, T_{\mathrm{tp}}\left(\mathrm{n}-\mathrm{D}_{2}\right)=18.73 \mathrm{~K}$, and
$c(\mathrm{HD})=0.0075$ into eq (6) yields a temperature shift of $\Delta T_{\mathrm{tp}}=-0.016 \mathrm{~K}$.

There is another possible source of systematic error. We noted earlier that our melting data are actually observations of the "first-freeze" behavior of the material. Grenier and White [35] similarly measured "firstfreeze" temperatures of deuterium. But, they also measured melting temperatures of samples that contained larger relative fractions of the solid phase, and for these they reported differences of "a few millikelvin." The effect was attributed to variations of the ortho-para compositions of the solid and liquid phases. While this suggestion may be true, we feel the data are insufficient to warrant any firm conclusions. Recall that our direct measurements of the melting curve slope (labeled " $3 a$ " in Fig. 2) gave results that are consistent with those derived from the differences of consecutive "firstfreeze" melting pressures. In our experiment, the meiting characteristics were affected little by the relative phase composition of the sample.

In view of these uncertainties, we feel that a triplepoint temperature of $18.723 \pm 0.010 \mathrm{~K}$ for pure $\mathrm{n}-\mathrm{D}_{2}$ is the most reasonable estimate from our measurements. If this value is used in eq (3), the calculated $P_{\mathrm{m}}$ values will have been corrected for the HD impurity.

\subsection{Liquid Isothermal Compressibility}

The isothermal compressibility $\beta$ is calculated from the measured change in the sample pressure $\Delta P_{\mathrm{u}}$ that results when the lower chamber pressure is changed by an amount $\Delta P_{1}$ at constant temperature. The expression used in this calculation

$$
\beta \equiv-\frac{1}{V}\left(\frac{\partial V}{\partial P}\right)_{r}=\frac{1}{\bar{V}_{\mathrm{a}}}\left[S_{\mathrm{u}}+S_{1}\left(1-\frac{\Delta P_{1}}{\Delta P_{u}}\right)\right]
$$

is easily derived from eq (1). In this equation, $\bar{V}_{\mathrm{u}}$ represents the average sample volume for the measurement. For small changes in $P_{1}$ and $P_{\nu}$, the sensitivity factors $S_{\|}$ and $S_{1}$ may be taken directly from the calibration curves. However, to obtain more precise compressibilities for the liquid, we chose to measure over larger pressure intervals. This procedure requires the sensitivity parameters in eq (7) to consist of rather complicated weighted averages of the measured $S_{\mathrm{u}}$ and $S_{1}$ functions.

A plot of the liquid isothermal compressibility measurements along three isotherms is shown in figure 4. The values obtained by extrapolating eight isotherms to the melting curve are represented by circles in the plot. The estimated accuracy is about $\pm 1 \%$.

The functional behavior of these data suggests that we consider a liquid equation of state of the form: 


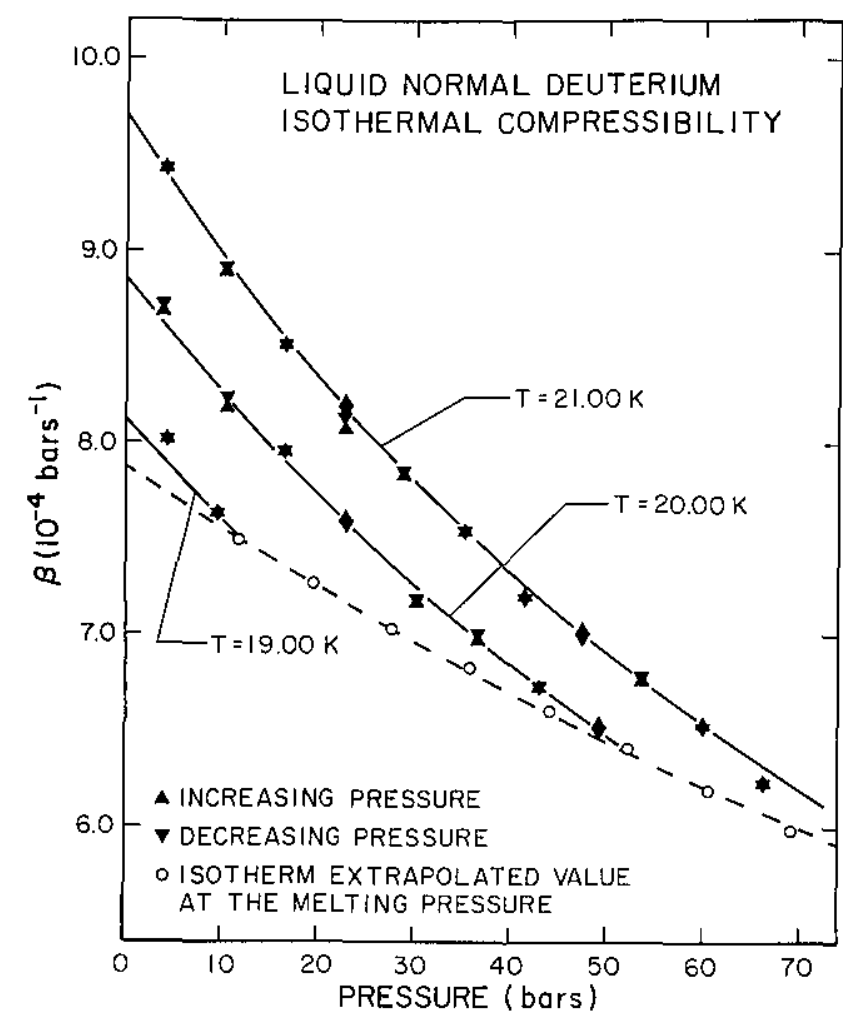

Figure 4-Isothermal compressibility data for liquid n- $D_{2}$. Solid curves are isotherms calculated from eq (9) and the fitting constants listed in table 1 . The dashed curve represents eq (9) evaluated along the melting line.

$$
\begin{gathered}
V(T, P)=V_{0}(T)\left[P+P_{0}(T)\right]^{-a} \\
V_{0}(T)=\exp \left[b_{0}+b_{1}\left(T-T_{\mathrm{tp}}\right)+b_{2}\left(T-T_{\mathrm{tp}}\right)^{2}\right. \\
\left.+b_{3}\left(T-T_{\mathrm{tp}}\right)^{3}\right] \\
P_{0}(T)=c_{0}+c_{1}\left(T-T_{\mathrm{tp}}\right)+c_{2}\left(T-T_{\mathrm{tp}}\right)^{2}
\end{gathered}
$$

where $T_{\mathrm{tp}}=18.7067 \mathrm{~K}$, and $a$ and the subscripted letters $b$ and $c$ are all constants to be determined by fitting eq (8) and their derivative functions to the experimental data.

For the isothermal compressibility, we obtain the following relationship:

$$
\beta(T, P) \equiv-\left(\frac{\partial \ln V}{\partial P}\right)_{T}=\frac{a}{P+P_{0}(T)}
$$

A fit of eq (9) to the compressibility data gives the numerical values for the constants $a, c_{0}, c_{1}$, and $c_{2}$ listed in table 2. In figure 4, the smooth curves depicted for three isotherms and the liquid compressibility along the melting line were calculated from eq (9) and these constant values.

To our knowledge, there are no published compressibility data in this temperature and pressure range
Table 2. Coefficients to eqs (8), (9), and (11) that describe the equation of state for the liquid.

$\begin{array}{lc}a=0.12 & c_{0}=152.0 \\ b_{0}=3.7408 & c_{1}=-13.2 \\ b_{1}=0.0017 & c_{2}=0.50 \\ b_{2}=0.00028 & \\ b_{3}=0.00001 & \end{array}$

that could be used for direct comparison. But, there have been several measurements of liquid molar volume at pressure and some estimates of compressibilities can be calculated from these by taking first differences. Bartholome [2] published volume data along three isotherms between $19.5 \mathrm{~K}$ and $21.0 \mathrm{~K}$. Compressibilities obtained from Bartholome's raw data are too scattered to allow any meaningful comparison, but those calculated from his smooth curves show qualitatively correct pressure and temperature dependences. Quantitatively, the agreement with our results is less satisfactory; compressibilities derived from the earlier work typically lie 10 to $14 \%$ higher than those calculated from eq (9).

Friedman et al.[6] published molar volume data for liquid normal deuterium at seven temperatures between 20.3 and $38.0 \mathrm{~K}$ and at pressures to $100 \mathrm{bar}$. Their volume measurements along the $20.33 \mathrm{~K}$ and $20.31 \mathrm{~K}$ isotherms were shown to agree reasonably well with the earlier but less precise Bartholome [2] data. Compressibilities derived from the Friedman $20.33 \mathrm{~K}$ data are in $\pm 1 \%$ agreement with values found from eq (9). Those calculated from their isotherms at $20.31 \mathrm{~K}$ and $23.52 \mathrm{~K}$ scatter about our fitted smooth curves with an average absolute deviation of about $6 \%$ and $4 \%$, respectively.

\subsection{Liquid Thermal Expansivity}

The isobaric thermal expansion coefficient $\alpha$ is calculated from the measured change in the lower-cell pressure $\Delta P_{1}$ that is required to maintain a constant sample pressure $P_{\mathrm{u}}$ when the temperature of the system is changed by an amount $\Delta T$. From eq (1) it follows that

$$
\alpha \equiv \frac{1}{V}\left(\frac{\partial V}{\partial T}\right)_{P}=-\frac{S_{1} \Delta P_{1}}{\bar{V}_{\mathrm{u}} \Delta T}-\frac{S_{\mathrm{u}}}{\bar{V}_{\mathrm{u}}}\left(\frac{\mathrm{d} P_{\mathrm{b}}}{\mathrm{d} T}\right)
$$

where $\vec{V}_{\mathrm{u}}$ is again the average sample volume for the measurement, and $\mathrm{d} P_{\mathrm{b}} / \mathrm{d} T$ is the temperature derivative of the liquid $\mathrm{p}-\mathrm{H}_{2}$ vapor pressure calculated from eq (2).

Figure 5 is a plot of the liquid expansion data obtained at six different pressures. We estimate the experimental uncertainty for these measurements to be about \pm 1 or $2 \%$. The data precision was limited to some extent by the temperature measurements but mostly by a slight 


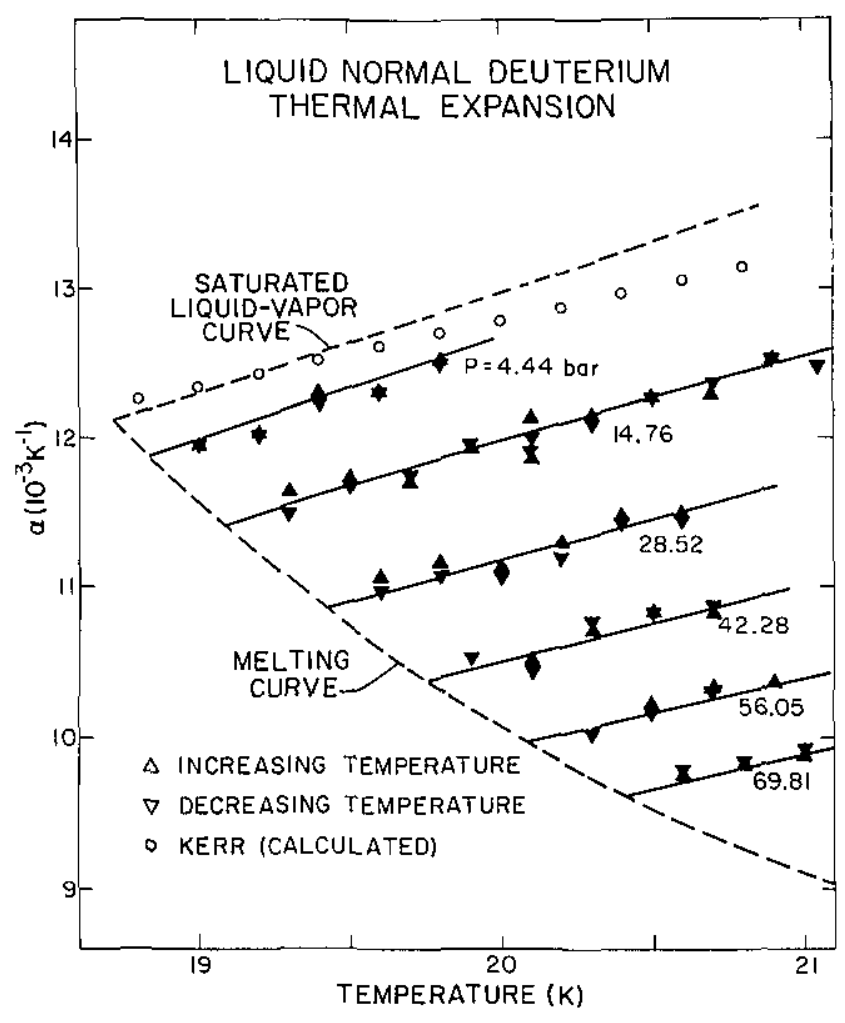

Figure 5 - Thermal expansion data for liquid $n-D_{2}$. Solid curves were calculated from eq (11) and the fitting constants listed in table 1. The dashed curve represents eq (11) evaluated at saturated liquidvapor pressures and melting as indicated. Open circles are calculated values from the fitted equation by Kerr [5].

nonlinearity in the pressure readout of the TI-144 bourdon gauge used for measuring $P_{1}$. Occasionally some hysteresis effects were observed in the deflection of the cell diaphragm although these only occurred for the initial points in either warming or cooling series of measurements. Errors of about $6 \%$ were observed for such artifacts, and these few data were discarded.

Differentiation of eq (8) yields the following expression for the thermal expansivity:

$$
\begin{aligned}
\alpha(T, P) & =b_{1}+2 b_{2}\left(T-T_{\mathrm{tp}}\right)+3 b_{3}\left(T-T_{\mathrm{tp}}\right)^{2} \\
& -\beta(T, P)\left[c_{1}+2 c_{2}\left(T-T_{\mathrm{tp}}\right)\right]
\end{aligned}
$$

where $T_{\mathrm{tp}}=18.7067$ and $\beta(T, P)$ is calculated from eq (9). The constants $a$ and the subscripted $c$ 's are those determined from the compressibility measurements. These constraints assure that the identity

$$
\left(\frac{\partial \alpha}{\partial P}\right)_{T} \equiv-\left(\frac{\partial \beta}{\partial T}\right)_{P}
$$

holds and, thereby, provide a condition of internal con- sistency that is automatically met if the expansivities can be fitted to eq (11) by adjusting only $b_{1}, b_{2}$ and $b_{3}$.

Equation (11) was fitted to the thermal expansivity data to fix the values in table 2 for the three additional constants. Smooth curves corresponding to the pressures of the six experimental isobars were calculated from eq (11) and are plotted with the data. The dashed lines in figure 5 represent the values along the vaporpressure and melting curves as indicated.

As before with the compressibilities, we found our thermal expansivity measurements to be the only explicit data in this range. Here again, we have derived estimates from the smooth curves that were fitted to independent measurements of the molar volume [2,5].

Kerr [5] measured the molar volume of the liquid along the vapor pressure curve. He combined his results with those of Clusius and Bartholome [1] and fitted them to a quadratic function of temperature. We calculated thermal expansion coefficients from this function by differentiation. The slight correction to constant pressure was made with eq (9) and Grilly's [23] vapor pressure function. The results of this calculation, included in figure 5 as the series of open circles, agree reasonably well with eq (11) near $19 \mathrm{~K}$ but deviate systematically with temperature to more than $2 \%$ at $20.5 \mathrm{~K}$.

Bartholome [2] presented measurements of the liquid molar volume as a function of pressure along three isotherms. Again, we consider the smooth curves that he fit to his raw data. The differences between these isotherms at constant pressure were taken to calculate average thermal expansivities in the two temperature intervals. At low pressures there is satisfactory agreement with our data. (Here, the consistency is reasonable if it is recalled that Bartholome calibrated the volume of his pressure cell along the vapor curve against the molar volume data of Clusius and Bartholome. The latter measurements were included by Kerr [5] in his quadratic fit, and thermal expansion coefficients derived from this function were shown to correspond closely to our extrapolated measurements.) However, with increasing pressure, the average thermal expansion coefficients calculated from Bartholome's data decrease much more rapidly than ours do. At 70 bars they fall to about $50 \%$ of our results.

\subsection{Liquid Molar Volume}

Data for $V_{\mathrm{lm}}$, the liquid molar volume along the melting curve, were obtained by a two-step operation. First, the volume of a sealed liquid sample was measured at a given temperature and two-phase pressure $P_{\mathrm{u}}$. The sample quantity was then determined by expanding the gas into a standard volume at room temperature. The latter measurement followed essentially the same procedure 
as that used for the calibration measurements of the cell volume and pressure-sensitivity factors.

The results are listed in table 3 . We estimate their accuracy and precision to be about \pm 0.035 and \pm 0.003 $\mathrm{cm}^{3} /$ mole, respectively. For all of the measurements except one, the data were taken within about $6 \mathrm{~h}$ from the time of the sample loading. The point at $T_{\mathrm{m}}=19.000_{8}$ $\mathrm{K}$ was obtained from a sample that had been loaded and held at that temperature for approximately $17 \mathrm{~h}$ before the measurement.

The results are plotted together as a function of temperature in figure 6. Also included in the figure are three points that Woolley et al. [4] had compiled from earlier work $[1,2]$. The liquid molar volume measurements of

Table 3. Measured liquid molar volumes along the' melting curve.

\begin{tabular}{cc}
\hline$T_{\mathrm{m}}(\mathrm{K})$ & $V_{\mathrm{lm}}\left(\mathrm{cm}^{3} /\right.$ mole $)$ \\
\hline $18.830_{8}$ & $23.00_{4}$ \\
$19.000_{8}$ & $22.93_{0}$ \\
$19.201_{0}$ & $22.84_{6}$ \\
$19.403_{0}$ & $22.76_{4}$ \\
$19.604_{3}$ & $22.68_{4}$ \\
$19.805_{5}$ & $22.60_{5}$ \\
$20.008_{0}$ & $22.53_{2}$ \\
$20.204_{5}$ & $22.45_{6}$ \\
$20.408_{5}$ & $22.38_{6}$ \\
\hline
\end{tabular}

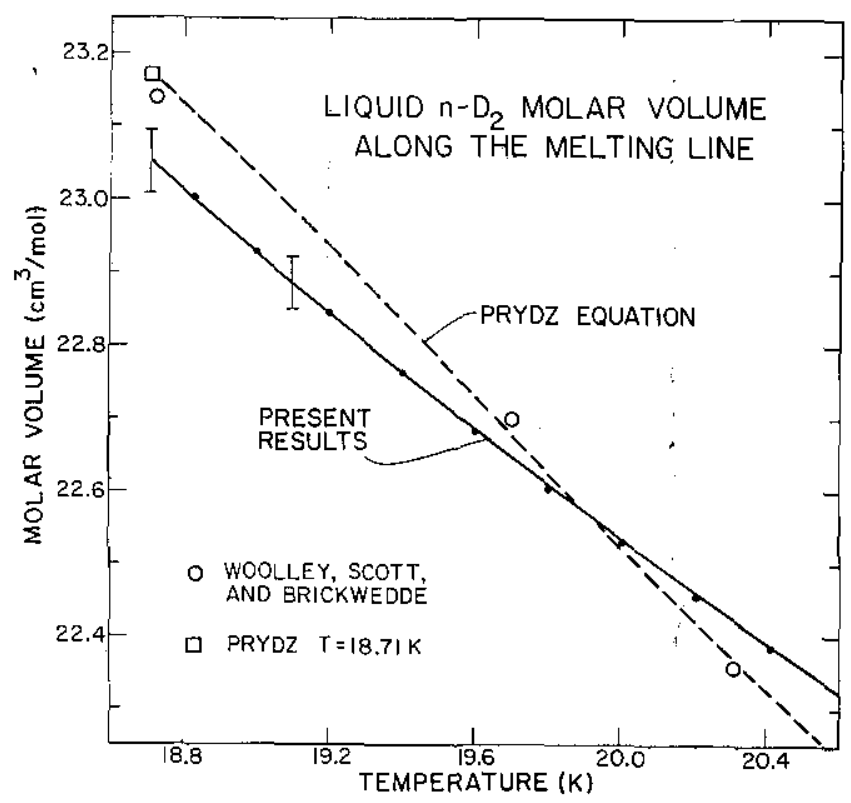

Figure 6-Measurements of the molar volume of liquid n- $\mathrm{D}_{2}$ at melting. The solid curve was calculated from eq (8) at the temperatures and pressures prescribed by eq (3). $A \pm 0.15 \%$ error is indicated for these results. The liquid density function of Prydz [7] was evaluated at the same points and the results are represented in the plot by the dashed curve. Also included are three points that were tabulated by Woolley et al. [4].
Bartholome were discussed in the two previous sections. To obtain the points at 19.70 and $20.31 \mathrm{~K}$, Woolley evaluated Bartholome's smoothed functions at the temperatures and pressures prescribed by the melting curve of Clusius and Bartholome [1]. The triple-point value, $23.14 \mathrm{~cm}^{3} /$ mole, was taken directly from the work of Clusius and Bartholome [1].

Prydz [7] used these and other earlier data to fit an equation of state for liquid normal deuterium. We evaluated the Prydz density function at temperatures and pressures corresponding to our observed melting line. The resulting curve, which is also plotted in figure 6 , adequately represents the earlier data. However, our measurements indicate that the liquid compression along the melting curve is not nearly as great as the Prydz function would suggest.

The liquid molar volume data were taken with the parameters $a, b_{1}, b_{2}, b_{3}, c_{0}, c_{1}$, and $c_{2}$ in table 2 to fix the remaining constant $b_{0}$. With the full set of fitting parameters, eq (8) can be used to calculate liquid $n-D_{2}$ molar volumes for any temperature and pressure within or even somewhat beyond the ranges of the measurements. The curve depicted in figure 6 was calculated along the melting line defined by eq (3). The average absolute deviation of the measured data from the calculated curve is $0.0026 \mathrm{~cm}^{3} / \mathrm{mole}$, which is comparable to the estimated precision of the measurements.

We have shown in this and in the two preceding sections that the isothermal compressibility, thermal expansion, and molar volume data for the liquid are all well described by a single empirical equation of state. This is a sufficient demonstration of the internal consistency of these measurements. It remains now to compare the predictions of this equation with independent data, but first some comment should be given about the effects of sample contamination.

Our volume data and their representation, eq (8), were presented without any correction for impurity effects. The choice is somewhat arbitrary as to whether or not these should be formally included. Some of the existing data were given as corrected values $[5,36]$, but others $[1,2,6]$ were published without corrections as we have done. The effects are not entirely negligible. For our case, the only significant impurity was the $0.75 \%$ HD discussed above. If we assume the volume correction is proportional to the HD concentration and to the difference between the molar volumes of HD and $D_{2}$, we have

$$
\Delta V_{1}=c(\mathrm{HD})\left[V_{1}(\mathrm{HD})-V_{1}\left(\mathrm{n}-\mathrm{D}_{2}\right)\right]
$$

The most recent density measurements on HD are those of Rudenko and Slyusar' [37], which extend from 16.6 to $35.5 \mathrm{~K}$ along the vapor curve. These data, combined 
with extrapolated values from eq (8), yield a volume difference of about $2 \mathrm{~cm}^{3} /$ mole. The correction to eq (8) is then toward smaller values by an amount 0.015 $\mathrm{cm}^{3} /$ mole. With the possible exception of some of the earliest work [1,2], the extent of impurity effects in previous data is comparable to that estimated for our own.

Bartholome [2] published the earliest measurements of the molar volume of $n-D_{2}$ at pressure. Bartholome's raw data scatter about the fitted smooth curves with an average absolute deviation of $0.025 \mathrm{~cm}^{3} /$ mole. The precision of these measurements is, therefore, roughly an order of magnitude less than that typical of more recent work $[5,6]$. The average absolute deviation of Bartholome's data from eq (8) is $0.073,0.035$ and 0.058 $\mathrm{cm}^{3} /$ mole for the isotherms at $19.723,20.346$ and 21.032 K (IPTS-68), respectively. Although these differences are probably no larger than the combined error for the two experiments, the earlier data show significant systematic deviations from eq (8). As has been mentioned the compressibilities derived from Bartholome's work are larger than our measurements by 10 and $14 \%$ at $T=20.346$ and $21.032 \mathrm{~K}$ (IPTS-68), respectively. The discrepancies may be the result of sample contamination which we shall discuss later in more detail.

The later measurements by Friedman et al. [6] provide us with a more critical basis for comparison. Of particular interest here are their tabulated results along the isotherms at 20.31 and $20.33 \mathrm{~K}$. We plotted these two sets of volume data as functions of pressure and found them to agree nearly identically. To compare these with our results, we next included the corresponding isotherm calculated from eq (8). The plot of the calculated values parallels the Friedman measurements but is uniformly lower in magnitude by about $0.05 \mathrm{~cm}^{3} / \mathrm{mole}$. This discrepancy is larger than our estimated error but probably smaller than the combined experimental uncertainties. Although Friedman et al. gave no explicit error limits, an accuracy comparable to our own $\left( \pm 0.035 \mathrm{~cm}^{3} / \mathrm{mole}\right)$ is reasonable. With this assumption the combined error is $\pm 0.07 \mathrm{~cm}^{3} /$ mole, and their volume measurements at $20.3 \mathrm{~K}$ are shown to agree with our equation of state. A similar comparison was made with their data at $23.52 \mathrm{~K}$. At this higher temperature, the difference between the earlier measurements and the values calculated from eq (8) is no greater than 0.035 $\mathrm{cm}^{3} /$ mole throughout the entire pressure range.

The Friedman data were presented without any explicit impurity corrections although the authors gave an impurity level of 0.2 at \% hydrogen (equivalent to $0.4 \%$ HD), roughly half the HD-concentration of our samples. This result seems improbable because most available deuterium has the same impurity as our sample, and they apparently made no special effort to purify their material. Ultimately, the accuracy of their impurity analysis (whether they had 0.4 or $0.8 \%$ HD) does not change the overall conclusion. The correspondence between their data and ours is not significantly affected by differences in contamination levels.

In a previous section, we mentioned that independent measurements of the liquid volume are available along the vapor pressure curve. These should provide useful comparisons, but because our lowest working pressures were between 4 and 5 bar, it is necessary to extrapolate our results somewhat. To do this, we calculated a molarvolume function of temperature by combining Grilly's [23] vapor-pressure equation for liquid $n-D_{2}$ with eq (8). A plot of this curve is shown in figure 7. Also included are the values Clusius and Bartholome [1] reported in the range 18.80 to $20.53 \mathrm{~K}$, the measurements Kerr [5] made between 19.5 and $24.5 \mathrm{~K}$, and the values found by extrapolating the data of Bartholome [2] to the vapor

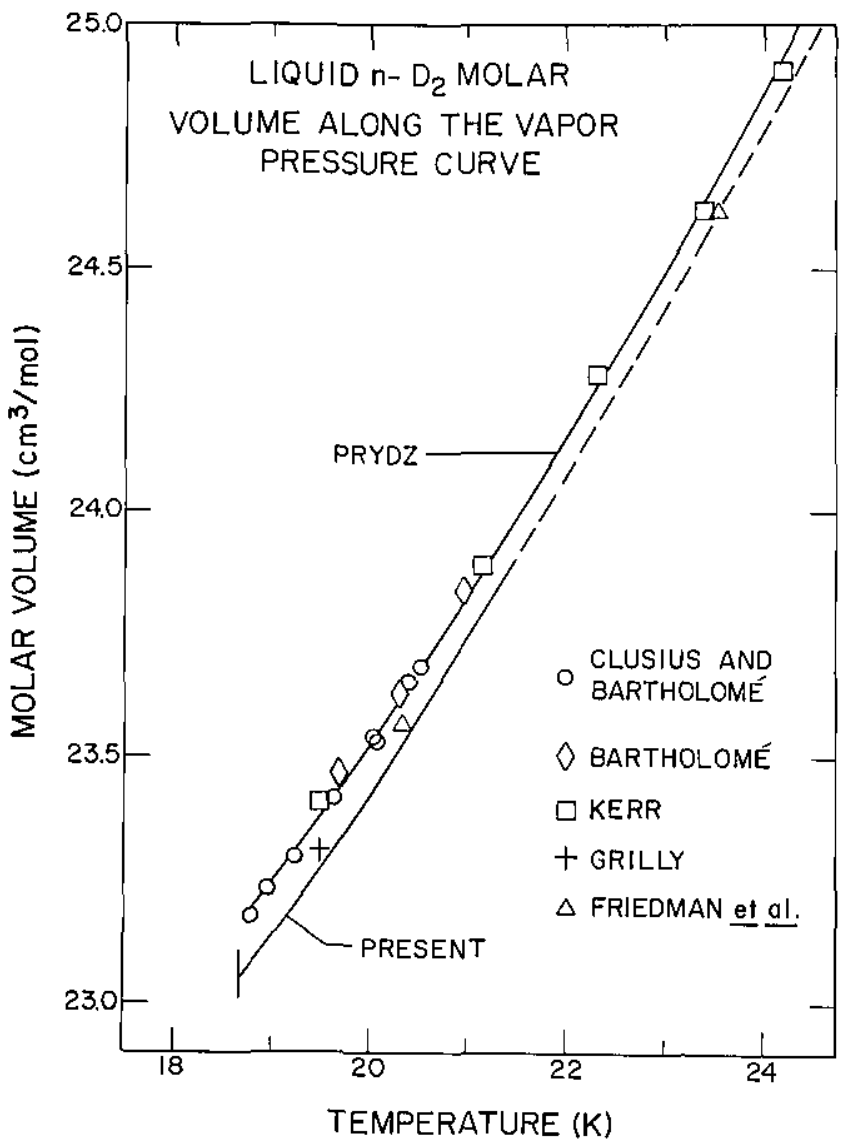

Figure 7-Existing molar volume data for liquid $n-D_{2}$ along the saturated vapor pressure curve. Direct measurements by Clusius and Bartholome [1], Grilly [36], and Kerr [5] are included with extrapolated values from the data of Bartholome [2] and Friedman et al. [6]. The solid curve through these data was calculated from the liquid equation of state of Prydz [7]. The lower curve was calculated from eq (8). The dashed portion represents an extrapolation beyond our measured temperature range. 
pressure curve. As figure 7 illustrates, these results are well described by the Prydz equation. However, throughout the plotted range, the earlier values are all consistently larger than those extrapolated from this work. In the interval 18.7 to $20.4 \mathrm{~K}$, the offset is approximately $0.5 \%$. About midway between the two curves are the extrapolated values from the data of Friedman et al. [6] and the direct measurement at $19.48 \mathrm{~K}$ by Grilly [36].

If each research had $\pm 0.15 \%$ uncertainty, our results agree with [6] and [36] but not with the others. The most serious discrepancy appears to be that between Kerr's data and ours. Kerr published five measurements of the molar volume between 19.5 and $24.2 \mathrm{~K}$. His results at 19.51 and $21.14 \mathrm{~K}$ are larger than corresponding values calculated from eq (8) by 0.13 and $0.10 \mathrm{~cm}^{3} / \mathrm{mole}$, respectively. Kerr stated that a mass spectrometric analysis of the sample showed it to contain 0.4 at \% hydrogen (equivalent to $0.8 \% \mathrm{HD}$ ) as the only detectable impurity. He corrected the data for impurities in the manner described above. If we compare our corrected data to his, the differences appear to be as large as 0.12 to $0.15 \mathrm{~cm}^{3} /$ mole. No error limits were quoted for the results Kerr presented, but if we assume an accuracy of $\pm 0.15 \%$, these data remain in substantial disagreement with our calculated values.

The source of the large discrepancy is not clear. We considered the possibility that some systematic error may have arisen from either of the volume calibrations at low temperature. Kerr calibrated his pycnometer with "pure hydrogen" and the data of Scott and Brickwedde [38]. On this point Kerr's report is somewhat ambiguous because Scott and Brickwedde included measurements on both liquid normal and para hydrogen. However, both sets of these earlier data extend along the saturated vapor pressure curve from 14 to $20.4 \mathrm{~K}$. They were obtained with the same experimental apparatus and, except for sample preparation, by the same procedure. We may reasonably assume that they are internally consistent. Therefore, regardless of the form of hydrogen that Kerr used, we can check his calibration against our own by comparing the respective p- $\mathrm{H}_{2}$ density standards.

It was not necessary for us to do this explicitly because Roder et al. [39] made this very comparison only a short time after the Goodwin data were published. To do this, Roder first fitted the Goodwin data to loworder polynomial expansions to represent both isotherms and isochores of the liquid density. These smoothed functions were then extrapolated to the saturated liquid-vapor pressure curve reported by Weber et al. [40]. Roder finally combined these "derived" data in the range 17.0 to $33.0 \mathrm{~K}$ with the Scott and Brickwedde measurements and fitted the set to a single analytic function. They showed that deviations from the fitting function were of the same magnitude as the expected error of the data. This overall consistency is taken to establish the correspondence between our own volume calibration and that of Kerr's research. Whatever the source of the discrepancy is in the deuterium measurements, it does not arise from any disparity in the hydrogen densities used for the calibrations of the low-temperature sample volumes.

It would appear from figure 7 that Kerr's data agree with those of Clusius and Bartholome [1] and Bartholome [2]. The average deviation of the Clusius and Bartholome results from our extrapolated function is $0.10 \mathrm{~cm}^{3} /$ mole. But, there are several reasons to suspect that these earlier data are too large by roughly 0.05 $\mathrm{cm}^{3} /$ mole. The molar volume measurements that Clusius and Bartholome made were only part of a rather comprehensive thermodynamic study. In addition to these data and among others, they included measurements of the triple-point and, at one temperature, the saturated liquid-vapor pressure. This information is useful because the most likely occurring sample contaminants in the earlier work on deuterium are $\mathrm{H}_{2}$ and HD [33]. It is known that both impurities tend simultaneously to increase measured vapor pressures and to depress observed triple-point temperatures. From the results of these additional measurements it is argued [33] that the sample lot of deuterium used by Clusius and Bartholome was contaminated to higher levels than were judged in their report. Estimates of either $1.0 \% \mathrm{H}_{2}$ or $2.6 \%$ HD are proposed to explain the observed offsets in their results for the vapor pressure and triplepoint temperature. In either case, the corresponding values for $\Delta V_{1}$ calculated from eq (13) are approximately $0.05 \mathrm{~cm}^{3} /$ mole.

In the later high-pressure experiment [2], Bartholome calibrated the volume of his cell with both liquid hydrogen and liquid deuterium. Absolute volumes were calculated from the measured mole contents by using the density data of Onnes and Crommelin [42] for hydrogen and Clusius and Bartholome [1] for deuterium. The results obtained from the two calibrations reportedly agree to within $0.03 \%$. This is interesting because the Onnes and Crommelin data are known now to be high by about $0.05 \mathrm{~cm}^{3} /$ mole [38]; the agreement suggests that the deuterium results of Clusius and Bartholome (and Bartholome [2]) are also high by the same amount. The discrepancy is exactly that estimated above from considerations of impurity effects. If the Clusius and Bartholome data are corrected for this suspected contaminant, their measurements are seen to agree almost identically with the direct measurement of Grilly [36] 
and also with the extrapolated data of Friedman et al. [6]. For the Clusius and Bartholome measurements, an error limit of $\pm 0.04 \mathrm{~cm}^{3} / \mathrm{mole}$ is not an unreasonable estimate, given the combined uncertainties of the data and the impurity correction. If this limit is assumed, their results are seen to be consistent with our own data even after the $0.015 \mathrm{~cm}^{3} /$ mole impurity correction is applied to the latter.

To summarize this discussion, we have examined several series of measurements of the molar volume of liquid $n-D_{2}$. Some of these were direct measurements along the saturated liquid-vapor pressure curve $[1,5,36]$ while others were made along isotherms at pressure $[2,6]$. The individual results were compared directly when possible and by extrapolation when not. Generally, the values prescribed by our equation of state are lower than those obtained from earlier measurements. Comparisons with independent measurements of the molar volume show our work to be consistent with that of Friedman et al. [6], which are the more precise of the existing data at pressure. At the vapor-pressure line, we find that our extrapolations are consistent with the data of Clusius and Bartholome [1] if these are corrected for a substantial impurity effect. However, there is a significant difference between the molar volume measurements of Kerr [5] and corresponding values calculated from our equation of state. We examined the possibility that some error may have arisen from inconsistencies in the hydrogen density data used as standards in the volume calibrations, but we were unable to substantiate this hypothesis. The source of the discrepancy between Kerr's results and ours is unknown.

\subsection{Other Related Data}

Most of the previous discussion has involved direct comparisons of our data with those of others. There are also indirect data comparisons worth considering. One quantity that is often discussed is the isochoric temperature derivative of the pressure, $(\partial P / \partial T)_{V}$. An anal$y$ tic form for $(\partial P / \partial T)_{V}$ can be derived in either of two ways: eq (8a) can be inverted to express the pressure as a function of temperature and volume, and the desired function is obtained by differentiation. Or, the result can be calculated directly from the identity

$$
\left(\frac{\partial P}{\partial T}\right)_{V} \equiv \frac{\alpha}{\beta} \quad:
$$

and eqs (9) and (11). In either case, we estimate an uncertainty of $\pm 2 \%$ for this calculated quantity.

In figure 8 , we have plotted $(\partial P / \partial T)_{V}$ for our liquid data along the melting curve as a function of tem-

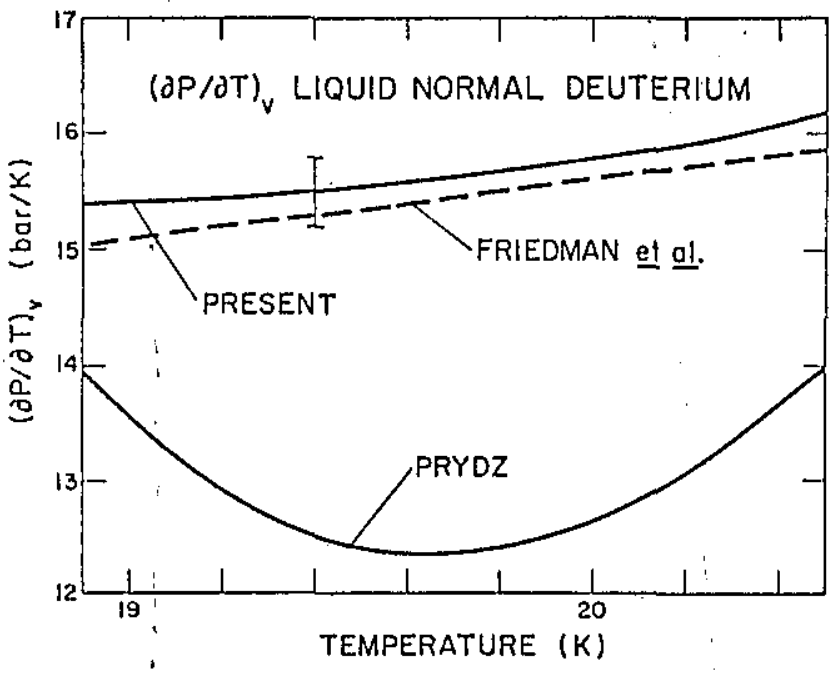

Figure 8-Plot of $(\partial P / \partial T)_{V}$ for liquid $\mathrm{n}-\mathrm{D}_{2}$ along the melting line defined by eq (3). The upper solid curve was.calculated from eq (14). The dashed curve was calculated from eq (15). The lower solid curve represents the empirical function for $(\partial P / \partial T)_{\rho}$ given by Prydz [7].

perature. For comparison, we have also included results calculated at the same temperatures and pressures from the $(\partial P / \partial T)_{\rho}$ function given by Prydz [7]. The curves show two obvious dissimilarities. First, they differ in magnitude by approximately $20 \%$. Second, the Prydz function shows a large positive curvature, whereas our $(\partial P / \partial T)_{V}$ results are more nearly constant with only a slight positive slope and curvature. The magnitude and functional behavior of our $(\partial P / \partial T)_{V}$ is consistent with that deduced by Friedman et al. [6] from their liquid PVT measurements. In particular, they found their data best described by the reciprocal volume relationship

$$
\left(\frac{\partial P}{\partial T}\right)_{V}=-14.3+\frac{674}{V_{1}}
$$

where the molar volume $V_{1}$ is taken in $\mathrm{cm}^{3} /$ mole and $(\partial P / \partial T)_{V}$ is given in bar/K. In figure 8, we have plotted the results obtained from eq (15) with the liquid molar volume data in table 3.

Thus far, we have only discussed equation-of-state data although these alone are not sufficient to describe the thermodynamic properties of a system. Supplementary thermal data are necessary for complete information. In connection with the present work, it is, therefore, interesting to briefly review the existing calorimetric data.

The earliest specific heat measurements on liquid $\mathrm{n}-\mathrm{D}_{2}$ were published by Clusius and Bartholome [1]. Their data, over the temperature range 19.4 to $21.7 \mathrm{~K}$, were later found to be about $8 \%$ lower than those of subsequent measurements [35,43]. Kerr et al. [43] con- 
cluded that some systematic error may have affected the earliest liquid results. Today, the liquid heat capacities of Clusius and Bartholome remain a matter of historic interest but are no longer given as preferred data [44].

Subsequent calorimetric measurements were reported by Kerr et al. [43] on o- $\mathrm{D}_{2}(2.2 \%$ para $)$ in the range 19.9 to $22.8 \mathrm{~K}$ and by Grenier and White [35] on $78.6 \%$ para at three temperatures between 19.9 and $22.1 \mathrm{~K}$. The results of these two experiments agree to within 1 or $2 \%$. Grenier and White considered this discrepancy reasonable in view of the uncertainties involved in the independent investigations. They further concluded that any effects of ortho-para composition are negligible in this temperature and pressure range.

Still later, Brouwer et al. [45] measured the specific heat of liquid $n-D_{2}$ as part of an extended study of the liquid phase separation properties of the $n-D_{2} / \mathrm{Ne}$ binary system. Their results for pure $\mathrm{n}-\mathrm{D}_{2}$ apparently extend over the entire range 20 to $30 \mathrm{~K}$ because they present a difference plot of the raw data from a fitted smooth curve. Curiously, Brouwer et al. did not explicitly include the analytic function in their publication, although they did tabulate values that were calculated from it at $0.2 \mathrm{~K}$ intervals between 24.0 and $27.4 \mathrm{~K}$.

In each of these four experiments, the actual measured quantity is the heat capacity along the saturated vapor pressure curve, which we denote as $C_{\text {sat }}$. From these data, the familiar isobaric quantity $C_{p}$ can be derived from the relationship:

$$
C_{p}=C_{\text {sat }}+T\left(\frac{\partial V}{\partial T}\right)_{P}\left(\frac{\mathrm{d} P}{\mathrm{~d} T}\right)_{v p}
$$

where $(\mathrm{d} P / \mathrm{d} T)_{v p}$ is the slope of the vapor pressure curve. The second term in eq (16) may be calculated from eqs (8a) and (11) and the temperature derivative of Grilly's [23] saturated liquid-vapor pressure function.

For temperatures below about $23 \mathrm{~K}$, the second term in eq (16) contributes less than $1 \%$ and could be neglected, but at higher temperatures there is a significant difference between $C_{p}$ and $C_{\text {sat. }}$. We, therefore, calculated $C_{p}$ explicitly for each of the later sets of measurements discussed above $[35,43,45]$. The results were then fitted to a quadratic function to yield

$$
\begin{gathered}
C_{p}\left(T, P_{v p}\right)=22.16+0.73(T-18.73) \\
+0.044(T-18.73)^{2} .
\end{gathered}
$$

We find that eq (17) reproduces the "corrected" Brouwer et al. [45] tabulated data to well within 1\%. The $C_{p}$ values corresponding to the Kerr et al. [43] measurements likewise fall in this range except, perhaps, for the highest temperature result, which may be lower by slightly more than $1 \%$. However, at roughly this same temperature, the Grenier and White [35] measurement is seen to fall about $2 \%$ higher than the smooth curve. We, therefore, postulate that eq (17) describes to within $\pm 1 \%$ the isobaric heat capacity for the liquid along the saturated vapor pressure curve from the triple point to $27 \mathrm{~K}$.

Corresponding values at higher pressures are calculated from

$$
C_{p}(T, P)=C_{p}\left(T, P_{v p}\right)-\int_{P_{v p}}^{P}\left(\partial^{2} V / \partial T^{2}\right)_{P} \mathrm{~d} P
$$

where a closed-form expression for the second term is derived from eq (8). Equation (18) illustrates that the qualitative effect of increasing pressure is to decrease $C_{p}$. For example, at $T=20.4 \mathrm{~K}$ and $P=70 \mathrm{bar}$, the calculated heat capacity is about $9 \%$ lower than its value at the same temperature on the vapor curve.

There are no independent measurements of $C_{p}$ at pressure that could be checked against eq (18) directly, but there are data for $C_{V}$, the specific heat at constant volume. Bartholome and Eucken [3] measured $C_{V}$ for liquid $\mathrm{n}-\mathrm{D}_{2}$ in the temperature range 19.5 to $23.5 \mathrm{~K}$. In principle, these results could be compared with $C_{\text {sat }}$ and our equation of state by calculating

$$
C_{V}(T, \mathrm{P})=C_{p}(T, P)-T V(T, P) \frac{\alpha^{2}(T, P)}{\beta(T, P)}
$$

where the first term on the right-hand-side is obtained from eqs (17) and (18) and the second from eqs (8a), (9), and (11).

There is, unfortunately, some ambiguity in the interpretation of the earlier results. To understand this, it is helpful to think of $C_{V}$ in terms of temperature and molar volume as the independent variables. The difficulty is then clear because Bartholome and Eucken did not explicitly include the sample density along with their temperature and specific-heat measurements. From the reported volume of the sample chamber $\left(3.5 \mathrm{~cm}^{3}\right)$ and the amount of material used for the liquid measurements ( 0.171 moles), we might infer the value $V_{1}=20.47$ $\mathrm{cm}^{3} /$ mole, but at this density we see from figure 6 that a sample could not exist in the liquid state over much of the quoted temperature range. We observe from eqs (3) and (8) that at $19.65 \mathrm{~K}$ the liquid molar volume must assume some value between $22.655 \mathrm{~cm}^{3} /$ mole at the melting line and $23.310 \mathrm{~cm}^{3} /$ mole at the saturated liquidvapor pressure.

Despite this ambiguity, there is some useful information available. The measurements of Bartholome and Eucken [3] are plotted in figure 9. If we assume these 


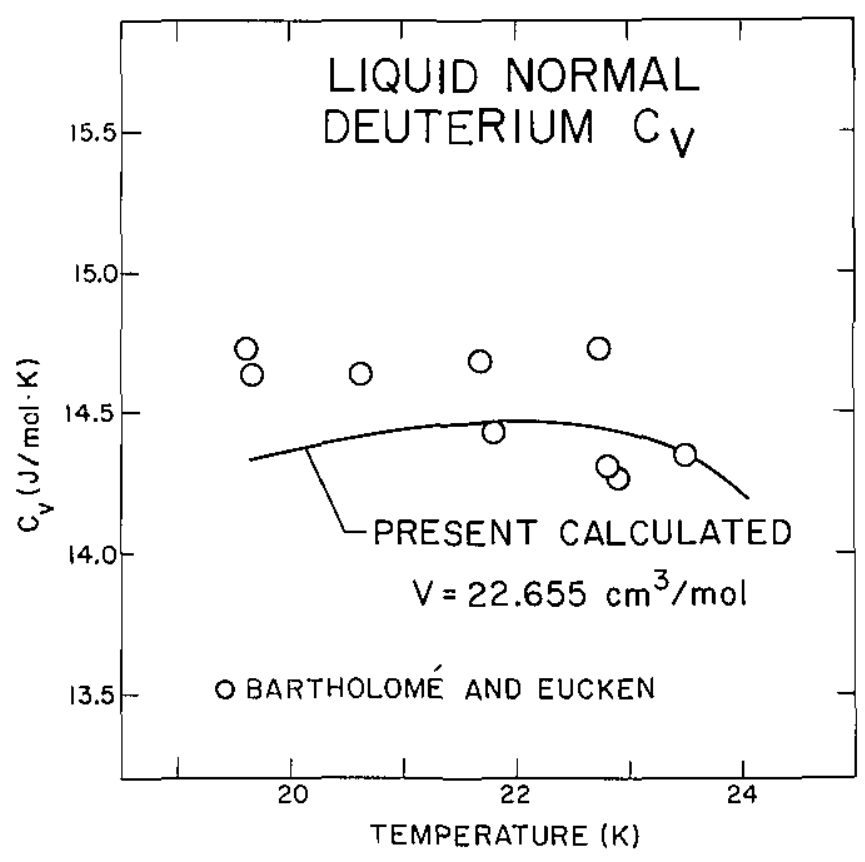

Figure 9-The isochoric heat capacity data of Bartholome and Eucken [3]. The solid curve was calculated from eq (19) for the liquid density at $19.65 \mathrm{~K}$ on the melting line.

data were terminated by the onset of freezing at $19.65 \mathrm{~K}$, a sample volume of $22.655 \mathrm{~cm}^{3} /$ mole is implied. The solid line plotted in figure 9 is the $C_{V}$ curve prescribed by eq (19) for this molar volume.

The calculated curve agrees reasonably well with the direct measurements. In the range 19.7 to $21.0 \mathrm{~K}$ the heat capacity results are reproduced to within $5 \%$. At higher temperatures the curve follows the measurements and seems to corroborate the observed weak temperature dependence that Bartholome and Eucken observed in deuterium. This agreement is somewhat fortuitous, however. The slope and curvature of the function defined by eq (19) is very sensitive to the values chosen for $b_{2}$ and $b_{3}$ in eq (11), much more so than are the results obtained from fitting our data. Below about $20 \mathrm{~K}$ the calculated $C_{V}$ remains little affected by the choice of these two parameters, but at temperatures near $24 \mathrm{~K}$, the results can vary by as much as $20 \%$.

Finally, we compare experimentally measured sound velocities in the liquid with those calculated from

$$
\frac{1}{u^{2}}=M\left(\frac{\beta(T, P)}{V(T, P)}-\frac{T \alpha^{2}(T, P)}{C_{P}(T, P)}\right)
$$

where $M=4.0282 \mathrm{gm} / \mathrm{mole}$ is the molecular weight of deuterium, and $u$ is the longitudinal sound velocity. The term in parentheses is calculated from eqs (8a), (9), (11), (17), and (18).
Bezuglyi and Minyafaev [46] published the first ultrasound velocity measurements on liquid $n-D_{2}$. Their results along the vapor curve at $T=19$ and $20 \mathrm{~K}$ are plotted in figure 10. Subsequent measurements by Wanner and Meyer [47] extend from the triple point both along the melting line to about $24 \mathrm{~K}$ and along the saturated-vapor-pressure curve to nearly $26 \mathrm{~K}$. Data from the lower portion of their measured temperature ranges are included in the plot. Typical error limits for these experiments are 0.5 to $1.0 \%$.

To compare with these direct measurements, we have included the results calculated from eq (20) and our own data. Sound velocities corresponding to our measured temperature range are indicated in figure 10 by the solid line segments. The dashed portions represent calculated

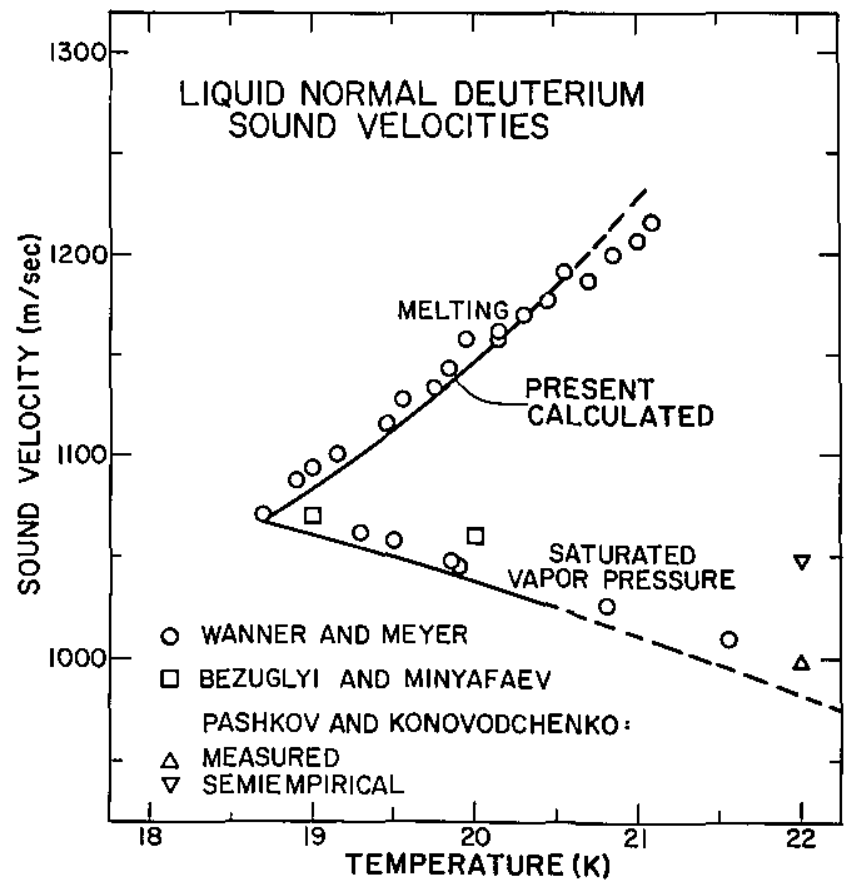

Figure $10-$ Liquid sound velocity measurements by Bezuglyi and Minyafaev [46], Wanner and Meyer [47], and Pashkov and Konovodchenko [48]. Also included in the plot is the semiempirical result that [48] derived from multistructure theory and the data of Prydz et al. [8]. Equation (20) was evaluated both along the saturated vapor pressure curve and at melting. The solid lines represent these results in the temperature range of the present experiment. Extrapolations of eq (20) are shown as the dashed portions.

values in the extrapolated regions. We estimate roughly $\pm 2 \%$ uncertainties for these results. In the range below $20.5 \mathrm{~K}$, the experimental measurements and the calculated curves agree. There is even a reasonable correspondence among the results along the vapor curve as high as $22 \mathrm{~K}$. However, along the melting line in the extrapolated range above $20.5 \mathrm{~K}$ there is a significant divergence. This discrepancy may indicate a systematic 
error in our measurements, but it may also be an artifact introduced by the particular functional form chosen to represent our equation of state.

Finally, we mention the work of Pashkov and Konovodchenko [48] who measured sound velocities at three temperatures: $22.00,26.00$, and $29.00 \mathrm{~K}$. At 22.00 $\mathrm{K}$ they obtained $998.8,1105.0$, and $1195.8 \mathrm{~m} / \mathrm{sec}$ at pressures of $0.605,50.66$, and $101.33 \mathrm{bar}$, respectively. Corresponding velocities calculated from eq (20) are 983, 1114 , and $1241 \mathrm{~m} / \mathrm{sec}$, respectively. At the two lower pressures there is satisfactory agreement between the measured and calculated values, but at 101.33 bar the difference is nearly $4 \%$ and is in the same sense as the divergence indicated at high temperatures along the melting line.

\subsection{Volume Change on Melting Heat of Fusion, and Solid Molar Volume}

The volume change on melting (freezing) $\Delta V_{\mathrm{m}}$ was measured by sealing the sample chamber with liquid $\mathrm{n}-\mathrm{D}_{2}$. At constant temperature, the lower cell chamber was first pressurized to freeze the sample and then depressurized to melt it. The volumes of the solid and liquid at the melting pressure were determined quite precisely from the abrupt slope discontinuities in $P_{\mathrm{u}}$ as observed in the calibrated capacitance measurements. The results presented in table 4 are expressed as $\Delta V_{\mathrm{m}} / V_{\mathrm{lm}}$, the volume change normalized to the liquid molar volume at the melting pressure. An uncertainty of about \pm 0.0005 is estimated for these data.

Equation (8) was used to calculate $\Delta V_{\mathrm{m}}$, the absolute volume changes. The results are plotted in figure 11 together with the single value, $2.66 \mathrm{~cm}^{3} /$ mole, that Clusius and Bartholome [1] quoted for the triple point. Bartholome [2] published the only other direct measurements at these temperatures. His average values, 2.16 and $1.98 \mathrm{~cm}^{3} /$ mole at 20.31 and $20.97 \mathrm{~K}$ respectively, are much smaller than our data. The $\Delta V_{\mathrm{m}}$ measurements by Liebenberg et al. [10] were carried out in a much higher range, 75 to $164 \mathrm{~K}$. The results were

Table 4. Measured volume changes on melting (freezing).

\begin{tabular}{lcc}
\hline \hline$T_{\mathrm{m}}(\mathrm{K})$ & $\Delta V_{\mathrm{m}} / V_{\operatorname{lm}}($ freezing $)$ & $\Delta V_{\mathrm{m}} / V_{\mathrm{lm}}$ (melting) \\
\hline 18.83 & 0.1171 & 0.1171 \\
19.00 & 0.1156 & 0.1156 \\
19.20 & 0.1144 & 0.1146 \\
19.40 & 0.1128 & 0.1131 \\
19.60 & 0.1120 & 0.1121 \\
19.80 & 0.1111 & 0.1112 \\
20.00 & 0.1099 & 0.1102 \\
20.20 & 0.1090 & 0.1091 \\
20.40 & 0.1078 & 0.1081 \\
\hline
\end{tabular}

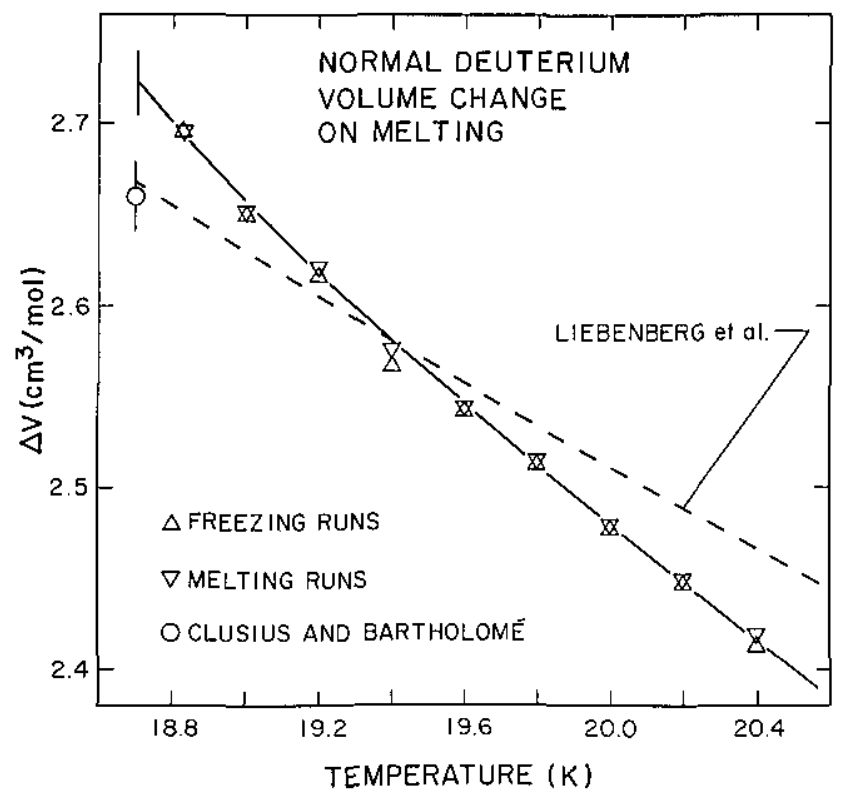

Figure 11-Measurements of the volume changes on melting. Included with these is the triple-point value derived by Clusius and Bartholome [1] from their measurement of the heat of fusion. The dashed line is an extrapolation of the empirical function given by Liebenberg et al. [10] to represent their high-temperature data.

fitted to an empirical function of temperature. The dashed curve in figure 11 represents this function extrapolated to the triple-point region. The agreement between Liebenberg's extrapolation and the ClusiusBartholome measurement is fortuitious.

The data presented here are relevant to some interesting questions first raised more than a decade ago. In 1973, Roder [49] speculated that hydrogen may exhibit a solid phase transformation near the melting curve at some temperature between 15 and $26 \mathrm{~K}$. The suggestion was made as an attempt to explain certain anomalies observed in solid molar volume [50] and heat capacity data. Independent measurements of the polarizibility $[30,51,52]$ and sound velocity $[46,47]$ in the solid phase also showed inconsistencies that seemed to corroborate his hypothesis.

At the same time that Roder's work appeared, Manzhelii et al. $[53,54]$ found that solid hydrogen undergoes an abrupt, but reversible, increase in density with increasing temperature at about $14 \mathrm{~K}$ and 30 bar. They attributed the effect to a solid phase transition. For a time, the proposed explanation drew some criticism $[13,14]$, but supporting evidence was later obtained from detailed measurements of the hydrogen melting line [24]. A small cusp-like singularity was observed near 14 $\mathrm{K}$ and interpreted as the intersection point of the solidsolid phase line. Mills [55] concluded that the equilibrium line of the solid hydrogen transformation must 
have negative slope, but the structure of the hightemperature form is still uncertain. X-ray diffraction studies at $16 \mathrm{~K}$ and 100 bar [56] suggest the change may be to an fcc structure, but the possibility of an isomorphic hep transformation has not been dismissed entirely [24].

Our volume-change measurements are relevant in this context because the solid phase transition is apparently not limited to pure p- $\mathrm{H}_{2}$ alone. Manzhelii et al. [54] and Bereznyak and Sheinina [24] discovered that the phenomenon is independent of the ortho-para concentration of hydrogen. Observed irregularities in the melting line of e- $\mathrm{D}_{2}$ [24] further suggest that similar transformations may be characteristic of the heavier isotopes as well. The effect is apparently also present in hydrogen-helium mixtures [57]. It is, therefore, reasonable to expect some indication of a solid phase transformation in the melting properties of $n-D_{2}$. Yet, as we mentioned earlier, there are no anomalies observed in our measurements of the melting curve. Nor do we observe any jump-like singularities in the data for the volume change on melting.

Manzhelii et al. [53,54] measured a density discontinuity of $0.15 \%$ in solid hydrogen. If a comparable difference in density were present between two solid phases of $n-D_{2}$, we would expect to see a step in the curve representing our results in figure 11 . The hightemperature solid phase should, by analogy, be more dense. The volume change from the liquid would be greater, and the step would be positive with increasing temperature. The discontinuity would occur at about $19 \mathrm{~K}$ and be about $0.03 \mathrm{~cm}^{3} /$ mole in magnitude. There is no obvious indication of such a step discontinuity in figure 11. Although it could be that the precision and density of our data are not sufficient to resolve the effect, it is difficult to dismiss the observed regularity of the melting pressure measurements. Our result does not directly contradict any existing data. At this time there is no evidence for a solid-solid phase transformation in solid $\mathrm{n}-\mathrm{D}_{2}$.

From measurements of the melting curve and the accompanying volume changes, the heat of fusion $\Delta H_{\mathrm{f}}$ of $\mathrm{n}-\mathrm{D}_{2}$ can be calculated with the Clausius-Clapeyron equation

$$
\Delta H_{\mathrm{f}}=T \frac{\mathrm{d} P_{\mathrm{m}}}{\mathrm{d} T_{\mathrm{m}}} \Delta V_{\mathrm{m}}
$$

Dwyer et al. [58] directly measured the heat of fusion of solid $\mathrm{p}-\mathrm{H}_{2}$ and found their results to be well described by a linear function of the melting pressure. Although they offered no explanation for this simple behavior, it suggests that we attempt a similar representation. A leastsquares fit to our calculated heats of fusion gives

$$
\Delta H_{\mathrm{f}}=197.22+0.179 P_{\mathrm{m}}
$$

where the melting pressure $P_{\mathrm{m}}$ is in bars and $\Delta H_{\mathrm{f}}$ is in $\mathrm{J} / \mathrm{mole}$. The average absolute deviation of the data from eq (22) is $0.17 \%$. No better fitting results are obtained by including quadratic or other high-order terms to the empirical function.

Calculated $\Delta H_{\mathrm{f}}$ are plotted as a function of pressure in figure 12. Included in the plot is the earlier direct result by Clusius and Bartholome [1], which is seen to be consistent with the present measurements. An extrapolation of eq (22) to the triple point yields a value of $197.25 \pm 0.40 \mathrm{~J} / \mathrm{mole}$. Kerr et al. [43] obtained $196.94 \pm 0.40 \mathrm{~J} / \mathrm{mole}$ for the heat of fusion of $\mathrm{o}-\mathrm{D}_{2}$ at the triple point. Measurements on $80 \%$ p- $\mathrm{D}_{2}$ by Grenier and White [35] give an average value of $197.41 \pm 0.40 \mathrm{~J} /$ mole. The general agreement among these independent data indicates that beyond the nominal error limits of $\pm 0.40 \mathrm{~J} / \mathrm{mole}$ there is no measurable dependence of the heat of fusion on ortho-para concentration in deuterium. The only inconsistent results are those presented by Bartholome [2] whose data on $n-D_{2}$ show $\Delta H_{f}$ decreasing with increasing temperature.

The molar volume $V_{\mathrm{sm}}$ of the solid along the melting line can be derived from the equation

$$
V_{\mathrm{sm}}=V_{\mathrm{lm}}\left(1-\frac{\Delta V_{\mathrm{m}}}{V_{\mathrm{lm}}}\right)
$$

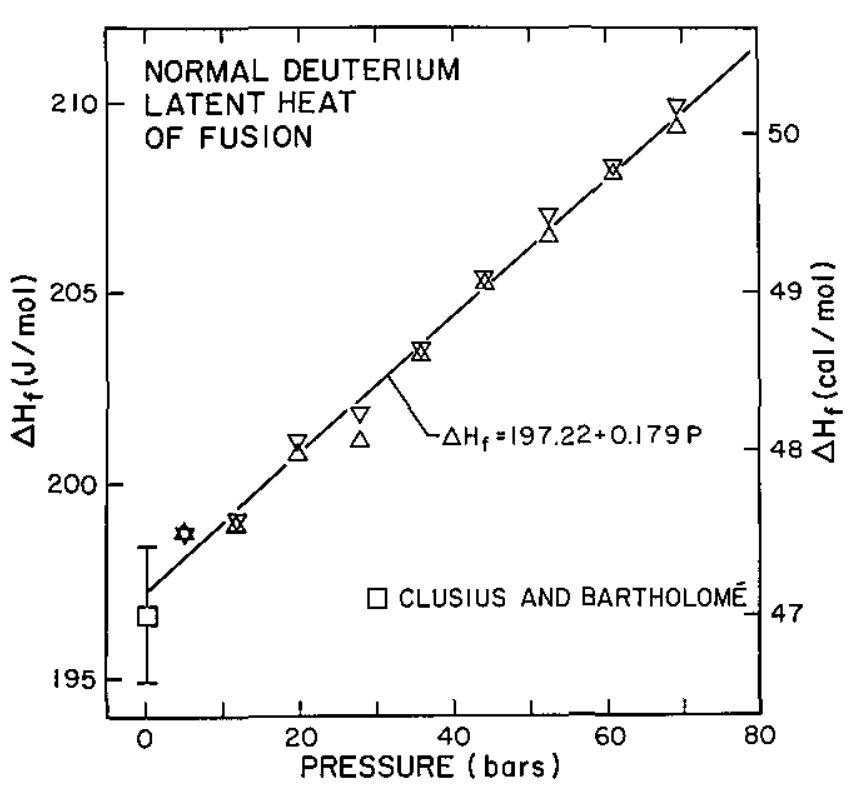

Figure 12-Data for the latent heat of fusion derived from eq (22). Included with these is the single direct measurement on $n-D_{2}$ by Clusius and Bartholome [1]. 
where the liquid molar volume $V_{\mathrm{lm}}$ at the same tem. perature and pressure is determined from eq (8). Calculated $V_{\mathrm{sm}}$ corresponding to the measured values of $\Delta V_{\mathrm{m}} / V_{1 \mathrm{~m}}$ in table 4 are plotted in figure 13. Also included in the plot are two points that Woolley et al. [4] derived from the work of Clusius and Bartholome [1] and Bartholome [2].

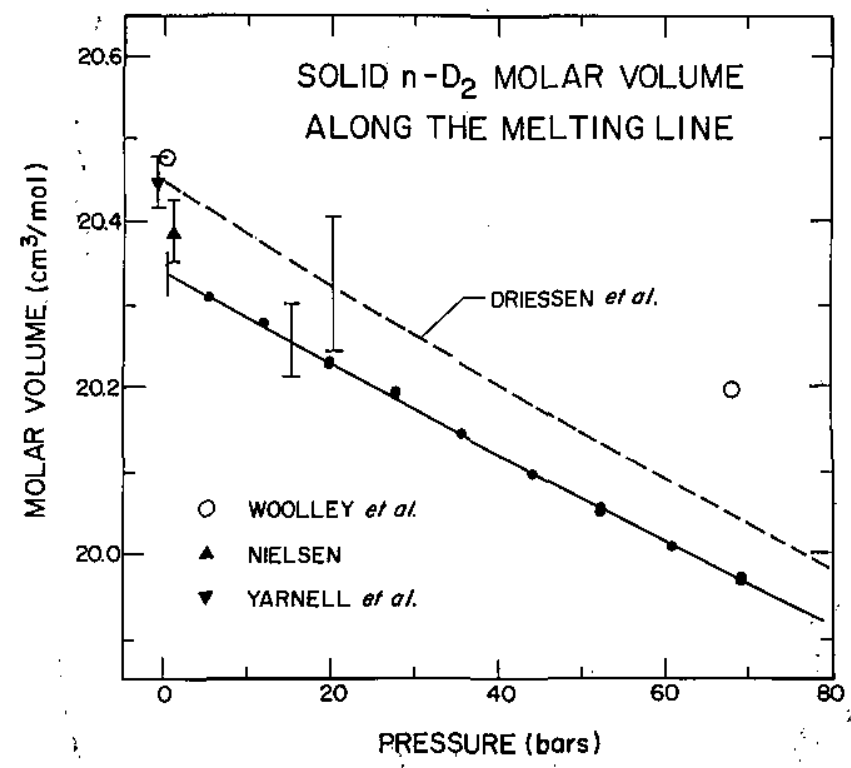

Figure 13-Data for the molar volume of solid n-D along the melting curve derived from eq (23). The dashed line was derived from the equation of state of Driessen et al. [14]. Also included are two points compiled by Woolley et al. [4] and two neutron diffraction results $[64,65]$.

The functional behavior of these data suggests a simple linear relationship. A least-squares fit gives

$$
V_{\mathrm{sm}}\left(P_{\mathrm{m}}\right)=20.337-0.0053 P_{\mathrm{m}}
$$

where $V_{\mathrm{sm}}\left(P_{\mathrm{m}}\right)$ is in $\mathrm{cm}^{3} /$ mole and the melting pressure $P_{\mathrm{m}}$ is in bars. The average absolute deviation of the data from eq (24) is $0.015 \%$. No improvement is obtained by including a quadratic term in the fitting polynomial. We estimate the accuracy of this expression to be about $\pm 0.04 \mathrm{~cm}^{3} / \mathrm{mole}$.

Within our range of measurements, eq (24) describes the data quite well, but it cannot be used reliably for extrapolation. A better representation is obtained by following some of the earlier work on p- $\mathrm{H}_{2}$. Dwyer et al. [59] first measured the molar volume of solid $\mathrm{p}-\mathrm{H}_{2}$ along the melting line at pressures up to 340 bar. Younglove [30] later corrected portions of these data and fit the solid densities to a linear function of melting temperature. A similar least-squares fit to the present data gives

$$
\rho_{\mathrm{sm}}\left(T_{\mathrm{m}}\right)=0.039133+0.0005362 T_{\mathrm{m}}
$$

where $\rho_{\mathrm{sm}}(T)$ is the density of solid n- $\mathrm{D}_{2}$ along the melting curve in moles $/ \mathrm{cm}^{3}$. Equations (24) and (25) both describe our measurements with comparable accuracy and precision, but eq (25) may provide a useful representation for the solid density to temperatures as high as

$27 \mathrm{~K}$. In the following discussion, we examine the consistency of eq (25), first with independent measurements on the low-pressure solid and then with various extrapolated data at higher pressures. We conclude this section with a discussion of the existing measurements of the dielectric constant of the solid and liquid phases.

An extrapolation of eq (25) to the measured triple point yields a density corresponding to $V_{\mathrm{sm}}\left(T_{\mathrm{tp}}=18.7067\right.$ $\mathrm{K})=20.342 \pm 0.04 \mathrm{~cm}^{3} /$ mole. It is possible to compare this value with the result of existing $\mathrm{x}$-ray [60], electron [61], and neutron scattering experiments [62-65] on the low-pressure solid.

For our discussion, the most important of this earlier work is the single-crystal neutron diffraction study of Nielsen [64]. These data include lattice parameter measurements on : $0-\mathrm{D}_{2}$ along its saturated vapor pressure curve from 5 to $18 \mathrm{~K}$. From the hcp lattice constants that Nielsen reported at $5 \mathrm{~K}$, we deduce a molar volume of $19.94 \mathrm{~cm}^{3} /$ mole. With increasing temperature, expansion of the material in the a and $\mathbf{c}$ directions was observed to be uniform within the limits of experimental uncertainty. Nielsen gave an analytical expression for the thermal expansion; from it we calculate for $0-D_{2}$ a molar volume of $20.41 \mathrm{~cm}^{3} /$ mole at $18.7 \mathrm{~K}$. Next, we apply the prescription of Driessen et al. [14] to calculate the small volume difference between ortho and normal deuterium at this temperature and density. The final result for solid $\mathrm{n}-\mathrm{D}_{2}, 20.39 \mathrm{~cm}^{3} /$ mole, is larger by about $0.05 \mathrm{~cm}^{3} /$ mole than that found by extrapolating eq (25). However, if the lattice measurements are accurate to $\pm 0.05 \%$, the uncertainty in the corresponding molar volume is $\pm 0.03 \mathrm{~cm}^{3} /$ mole. A small additional error is involved with the extrapolation to $18.7 \mathrm{~K}$. When these uncertainties are combined with those of the present study, the neutron diffraction result is shown to agree with our triple-point value.

Nielsen [64] published the only measurements on large single-crystals. The remaining work includes powder-diffraction data $[60,62,63,65]$ and one set of measurements on relatively thin samples grown on a cold substrate [61]. We have included a compilation of these results in table 5. All of the data were taken at temperatures lower than the triple point, but we have included corrections to the corresponding solid molar volumes of $n-D_{2}$ at $18.7 \mathrm{~K}$ in the manner described 
Table 5. Compilation of existing measurements of the molar volume of solid deuterium.

\begin{tabular}{|c|c|c|c|c|}
\hline References & $\begin{array}{l}\text { Sample } \\
\text { (\% para) }\end{array}$ & Temperature & $\begin{array}{c}\text { Measured } \\
\text { molar volume } \\
\left(\mathrm{cm}^{3} / \text { mole }\right)\end{array}$ & $\begin{array}{l}\text { Molar volume } \\
\text { corrected to } 18.7 \mathrm{~K}^{\mathrm{a}} \\
\left(\mathrm{cm}^{3} / \text { mole }\right)\end{array}$ \\
\hline $\begin{array}{l}\text { Mucker et al. (1965) [62] } \\
\text { neutron powder diffraction }\end{array}$ & $\begin{array}{r}33 \% \\
2 \%\end{array}$ & $\begin{array}{l}13 \mathrm{~K} \\
13 \mathrm{~K}\end{array}$ & $\begin{array}{l}20.08( \pm 0.12)^{b} \\
20.08( \pm 0.12)^{b}\end{array}$ & $\begin{array}{l}20.39 \pm 0.12 \\
20.41 \pm 0.12\end{array}$ \\
\hline $\begin{array}{l}\text { Schuch and Mills (1966) [60] } \\
\text { x-ray powder diffraction }\end{array}$ & $33-65 \%^{\mathrm{c}}$ & $1 . .5 \mathrm{~K}$ & $19.80 \pm 0.20^{d}$ & $20.28 \pm 0.20$ \\
\hline $\begin{array}{l}\text { Bostanjoglo and Kleinschmidt (1967) } \\
\text { [61] electron powder diffraction }\end{array}$ & $33 \%$ & $4.2 \mathrm{~K}$ & $19.90 \pm 0.11$ & $20.37 \pm 0.11$ \\
\hline $\begin{array}{l}\text { Mucker et al. (1968) [63] } \\
\text { neutron powder diffraction }\end{array}$ & $\begin{array}{r}3 \% \\
33 \% \\
63 \% \\
80 \% \\
80 \%\end{array}$ & $\begin{array}{r}12.9 \mathrm{~K} \\
10.2 \mathrm{~K} \\
2.0 \mathrm{~K} \\
10.5 \mathrm{~K} \\
4.2 \mathrm{~K}\end{array}$ & $\begin{array}{l}20.02( \pm 0.12)^{\mathrm{e}} \\
19.91( \pm 0.12)^{\mathrm{c}} \\
19.74( \pm 0.12)^{\mathrm{e}} \\
19.84( \pm 0.12)^{\mathrm{c}} \\
19.85( \pm 0.12)^{\mathrm{e}}\end{array}$ & $\begin{array}{l}20.33 \pm 0.12 \\
20.33 \pm 0.12 \\
20.32 \pm 0.12 \\
20.37 \pm 0.12 \\
20.46 \pm 0.12\end{array}$ \\
\hline $\begin{array}{l}\text { Nielsen (1973) [64] } \\
\text { single-crystal neutron diffraction }\end{array}$ & $2 \%$ & $5.0 \mathrm{~K}$ & $19.94( \pm 0.03)^{f}$ & $20.39 \pm 0.04$ \\
\hline $\begin{array}{l}\text { Yarnell et al. (1975) [65] } \\
\text { neutron powder diffraction }\end{array}$ & $2 \%$ & $4.2 \mathrm{~K}$ & $19.994 \pm 0.013$ & $20.45 \pm 0.03$ \\
\hline
\end{tabular}

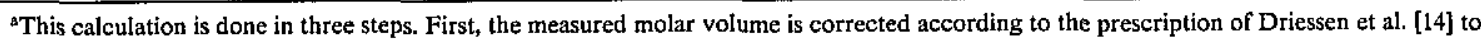
the value corresponding to zero percent para at the same temperature. Next, the temperature correction is made to $18.7 \mathrm{~K}$ using the analytic expression for the linear thermal expansion by Nielsen [64]. Finally, the o- $\mathrm{D}_{2}$ volume is corrected at $18.7 \mathrm{~K}$ to that of $\mathrm{n}-\mathrm{D}_{2}$ again according to reference [14]. We assume a error of $\pm 0.03 \mathrm{~cm}^{3} / \mathrm{mole}$ for this calculation. The accuracy limit in column 5 is taken as the square root of the sum of the squares of the error limit in column 4 and the assumed error in the temperature correction.

bEstimated error assumes $\pm 0.005 \hat{\AA}$ uncertainty in $a$ and \pm 0.005 in $\mathrm{c} / a$.

cNo trend was observed in the hcp molar volume with para concentration.

dWe set the uncertainty limit equal to the observed data scatter.

'The $\pm 0.6 \%$ volume error was calculated from the quoted $\pm 0.2 \%$ uncertainty in the nearest neighbor distance.

'The $\pm 0.15 \%$ volume error was calculated with an assumed $0.05 \%$ uncertainty for the lattice parameter measurements.
}

above. Generally, the corrected data are consistent with the extrapolated results of the present measurements. However, there are relatively large experimental uncertainties associated with most of the earlier work [60-63]. The data of Nielsen [64] and Yarnell et al. [65] are more precise. As we mentioned above, the solid molar volume obtained by extrapolating eq (25) to the triple point agrees with Nielsen's measurements, but it is 0.11 $\mathrm{cm}^{3} /$ mole lower than the corresponding value inferred from the work of Yarnell.

At higher pressures, the most instructive comparisons are made using the solid equations of state established by Driessen et al. $[13,14]$. This work was based in large part on their measurements of the isochores of solid $\mathrm{n}-\mathrm{H}_{2}$ and $\mathrm{n}-\mathrm{D}_{2}$ to pressures up to $2 \mathrm{kbar}$ at the melting line. The molar volumes were not measured directly but were determined by correlating the isochore data with existing measurements at $4.2 \mathrm{~K}$ for both $\mathrm{H}_{2}$ and $\mathrm{D}_{2}$ [12] and along the melting line for $\mathrm{H}_{2}$ [59]. The equations of state for $\mathrm{p}-\mathrm{H}_{2}$ and $\mathrm{o}-\mathrm{D}_{2}$ are presented in tabular form, and corrections to arbitrary ortho-para mixtures are accomplished by straightforward calculation.
A direct comparison of our data to those of Driessen et al. is not possible. Their lowest density isochore corresponds to $19.851 \mathrm{~cm}^{3} /$ mole, which is slightly beyond the range of our data. However, they did extrapolate their equation of state to low pressures. We interpolated their tabulated values to calculate the molar volume of $\mathrm{n}-\mathrm{D}_{2}$ at the temperatures and pressures corresponding to our melting line. The results are plotted in figure 13 . The $\pm 0.42 \%$ uncertainty was taken from figure 22 of their paper [14]. Our measurements agree with the extrapolated results of Driessen et al. within the combined error limits. However, we note that the most reliable of the low-pressure diffraction work $[64,65]$ is more nearly consistent with the extrapolated equation-of-state than it is with eq (25).

Driessen et al. quoted a value of $19.95 \mathrm{~cm}^{3} /$ mole for $\mathrm{o}-\mathrm{D}_{2}$ at zero temperature and pressure. This corresponds closely to the volume, $19.94 \pm 0.03 \mathrm{~cm}^{3} / \mathrm{mole}$, that Nielsen measured directly at $5 \mathrm{~K}$. According to the equation of state, this isochore should intersect the melting line at 90.7 bar. The corresponding melting temperature, $20.90 \mathrm{~K}$, was used in eq (25) to calculate an 
extrapolated density, and from this we obtain the molar volume $19.87 \pm 0.04 \mathrm{~cm}^{3} /$ mole. Nielsen's measurement corrected to the normal spin mixture at $20.90 \mathrm{~K}$ becomes $19.92 \pm 0.03 \mathrm{~cm}^{3} /$ mole. Again, the two results agree within experimental error.

In addition to his low-pressure data, Nielsen measured the lattice parameters of hcp o- $\mathrm{D}_{2}$ at $5 \mathrm{~K}$ and 275 bar. From these lattice data, we calculate a molar volume of $18.64 \pm 0.03 \mathrm{~cm}^{3} /$ mole, which is consistent with the Driessen et al. analysis at that temperature. (Existing $P \rightarrow V$ data at low temperatures [12] were fitted to the Birch equation. At this solid density, this equation yields a pressure of 271.5 bar.)

According to eq (19) of reference [14], the isochore at $18.64 \mathrm{~cm}^{3} /$ mole intersects the melting line at 422.5 bar. At this pressure the melting temperature, $27.45 \mathrm{~K}$, in eq (25) yields a molar volume of $18.57 \pm 0.04 \mathrm{~cm}^{3} / \mathrm{mole}$, which agrees with $18.63 \pm 0.03 \mathrm{~cm}^{3} /$ mole, the Nielsen volume corrected to $33 \%$ para at $27.5 \mathrm{~K}$. Equation (25) is thus seen to reproduce the extrapolated results of the neutron diffraction measurements within the combined limits of experimental error for the two experiments. This equation may, therefore, provide a useful representation for the solid density along the melting line to temperatures as high as $27 \mathrm{~K}$.

There have been a few measurements of the dielectric constant of solid [52] and liquid $[66,67] n-D_{2}$. It is instructive to discuss these results in terms of the molecular polarizability of the material. To do this, it is necessary to combine the dielectric data with independent measurements of the sample density. The polarizability $P_{E}$ can then be calculated from an expression such as the Clausius-Mossotti equation

$$
P_{E}=\frac{3 V}{4 \pi N_{\mathrm{a}}}\left(\frac{\epsilon-1}{\epsilon+2}\right)
$$

where $N_{\mathrm{a}}=6.0225 \times 10^{23} \mathrm{molecules} / \mathrm{mole}$ and $V$ is the molar volume.

We first apply eq (26) to the existing liquid data. Kogan et al. [66] measured the dielectric constant of liquid $\mathrm{n}-\mathrm{D}_{2}$ along the saturated vapor-pressure curve from the triple point to $20.4 \mathrm{~K}$. From their 12 data points and the corresponding liquid molar volumes computed from eq (8), we calculate an average value of $P_{E}=7.9371 \times 10^{-25}$ $\mathrm{cm}^{3}$ with a standard deviation of $0.0014 \times 10^{-25} \mathrm{~cm}^{3}$. This result agrees with those derived from similar data reported by Constable et al. [67] in the range 18.9 to 23.0 K. From their 22 measurements, we calculate an average of $7.9330 \times 10^{-25} \mathrm{~cm}^{3}$ with a standard deviation of $0.0077 \times 10^{-25} \mathrm{~cm}^{3}$.

To our knowledge, the only solid phase measurement on $n-D_{2}$ is that published by Wallace and Meyer [52].
Their value, $\epsilon=1.3324$, was obtained from a single sample under the melting pressure at $21.1 \mathrm{~K}$. The molar volume of the sample is calculated from eq (25) to be $V_{\mathrm{sm}}=19.825 \mathrm{~cm}^{3} / \mathrm{mole}$. These values substituted into eq (26) give $P_{E}=7.8388 \times 10^{-25} \mathrm{~cm}^{3}$ which is about $1.2 \%$ lower than the values derived from the liquid data.

The large difference between the derived polarizabilities for the solid and liquid phases is probably artificial. The melting pressure at $21.1 \mathrm{~K}$ is only $99.4 \mathrm{bar}$ according to eq (3). There should be no significant change in the liquid polarizability at this temperature over the limited pressure interval between the vapor and melting curves. Udovidchenko and Manzhelii [51] measured isothermal compressibilities of solid $\mathrm{p}-\mathrm{H}_{2}$ to pressures as high as 180 bar. They assumed a pressureindependent molecular polarizability in their data analysis. The results agree with the compressibilities that Driessen et al. [14] later found from their isochoric measurements.

Again, there is probably little if any difference between the molecular polarizabilities of the solid and liquid phases at $21.1 \mathrm{~K}$ on the melting line. Once more, our justification derives from independent work on $\mathrm{p}-\mathrm{H}_{2}$. Younglove [30] first measured the dielectric constants of solid and liquid $\mathrm{p}-\mathrm{H}_{2}$ along the melting line. Although, these data suggest a possible difference of $0.4 \%$, subsequent measurements by Wallace and Meyer [52] and Udovidchenko and Manzhelii [51] have not supported Younglove's findings. The later experiments obtained solid-and liquid-phase polarizabilities that are identical to within 0.1 to $0.2 \%$, an accuracy limit imposed by the available solid density data. The large apparent difference in the deuterium polarizabilities suggests to us that more careful measurements of the dielectric constant are required for both solid and liquid in this pressure range.

\subsection{Solid Thermal Expansivity and Isothermal Compressibility}

Measurement accuracies of solid-phase properties are severely constrained with the present technique. The problem derives from the nonplastic-flow characteristics of the solid and a rather sensitive dependence of the experimental results on the homogeneity of the sample density. For measurements of both thermal expansivity and isothermal compressibility, it is necessary to measure changes in the sample volume that result from unequal deflections of the upper and middle diaphragms of the pressure cell (see Fig. 1). Volume changes of the thin disk-shaped solid always introduce radial variations in the compression. As $P_{1}$ is increased, for example, the middle diaphragm is displaced upward, and the sample 
is compressed to a higher density at the center than around the periphery.

For liquids, the distortion of the sample geometry is no problem. Reasonably accurate results were even obtained earlier on solid helium [16,17], although hysteresis effects, particularly at the lowest temperatures indicate similar problems. With solid $n-D_{2}$, we cannot assume that our samples were free of internal strain even after several hours equilibration near the two-phase region. We observed that density gradients persisted in sufficient degree to preclude all but very approximate measurements of $\alpha$ and $\beta$ for the solid.

We tried a number of approaches to defeat the problem but could only obtain reproducible results by allowing long (2-10 h) relaxation periods with the solid at temperatures and pressures near the melting curve. Typically, in the melting pressure range 20 to $70 \mathrm{bar}$, the isothermal compressibilities decreased from about 3.8 to $2.0 \times 10^{-4} \mathrm{bar}^{-1}$ with increasing $P_{\mathrm{m}}$. Thermal expansivities were in the range 2.4 to $2.6 \times 10^{-3} \mathrm{~K}^{-1}$ for melting temperatures between 19.2 and $20.4 \mathrm{~K}$.

These results are somewhat inconsistent with our solid molar volume data. Consider the equation

$$
-\frac{1}{V_{\mathrm{sm}}}\left(\frac{\mathrm{d} V_{\mathrm{sm}}}{\mathrm{d} P_{\mathrm{m}}}\right)=\beta_{\mathrm{sat}}-\frac{\alpha_{\mathrm{sm}}}{\mathrm{d} P_{\mathrm{m}} / \mathrm{d} T_{\mathrm{m}}}
$$

where $\beta_{\mathrm{sm}}$ and $\alpha_{\mathrm{sm}}$ denote respectively the isothermal compressibility and thermal expansivity for the solid at melting. The quantity on the left-hand side of this equation represents a coefficient of compression for the solid along the melting curve. It can be calculated from the fitted curve of eq (24); the resulting values increase from $2.61 \times 10^{-4} \mathrm{bar}^{-1}$ at the triple point to $2.65 \times 10^{-4} \mathrm{bar}^{-1}$ at $70 \mathrm{bar}$. The average measured values over the pressure range 20 to 70 bar, $\beta_{\mathrm{sm}}=2.73 \times 10^{-4} \mathrm{bar}^{-1}$, $\alpha_{\mathrm{sm}}=2.56 \times 10^{-3} \mathrm{~K}^{-1}$, and $\mathrm{d} P_{\mathrm{m}} / \mathrm{d} T_{\mathrm{m}}=41.5$ bar $\mathrm{K}^{-1}$, yield the value $2.11 \times 10^{-4} \mathrm{bar}^{-1}$ for the right hand side of eq (27).

Estimates of the solid-phase properties are available from the Driessen et al. [14] equation of state. Although their tabulated equation is for $0-D_{2}$, the compressibility and thermal expansion coefficient for $n-D_{2}$ can be calculated from the information provided. In the temperature range 18.7 to $21.0 \mathrm{~K}$, the differences between the differential quantities of $o-D_{2}$ and $n-D_{2}$ are less than $1 \%$. At the triple point, we calculate a compressibility for solid $\mathrm{n}-\mathrm{D}_{2}$ of $4.28 \times 10^{-4} \mathrm{bar}^{-1}$ and a thermal expansion of about $5.3 \times 10^{-3} \mathrm{~K}^{-1}$. At $T_{\mathrm{m}}=20.5 \mathrm{~K}$, the corresponding values are about $12 \%$ lower.

An indirect estimate of the solid thermal expansivity can be made from some of the data that we have already discussed. Consider the temperature change of the liquid enthalpy along the melting curve

$$
\frac{\mathrm{d} H_{\mathrm{lm}}}{\mathrm{d} T_{\mathrm{m}}}=C_{P, \mathrm{~lm}}+V_{\mathrm{lm}} \frac{\mathrm{d} P_{\mathrm{m}}}{\mathrm{d} T_{\mathrm{m}}}\left(1-T_{\mathrm{m}} \alpha_{\mathrm{lm}}\right)
$$

In this expression $C_{P, \mathrm{Im}}$ denotes the isobaric heat capacity of the liquid at the melting line. The right-hand side of eq (28) can be evaluated from eqs (4), (8), (11), and (17). At the triple point we obtain $\mathrm{d} H_{\mathrm{lm}} / \mathrm{d} T_{\mathrm{m}}=91.5 \pm 1.0 \mathrm{~J} /$ mole $\mathrm{K}$.

The enthalpy of the solid at melting $H_{\mathrm{sm}}$ is equal to the liquid enthalpy minus the heat of fusion. We may, therefore, write

$$
\frac{\mathrm{d} H_{\mathrm{sm}}}{\mathrm{d} T_{\mathrm{m}}}=\frac{\mathrm{d} H_{1 \mathrm{~m}}}{\mathrm{~d} T_{\mathrm{m}}}-\frac{\mathrm{d}\left(\Delta H_{\mathrm{f}}\right)}{\mathrm{d} P_{\mathrm{m}}} \frac{\mathrm{d} P_{\mathrm{m}}}{\mathrm{d} T_{\mathrm{m}}}
$$

The second term evaluated from eqs (4) and (22) contributes an amount $7.0 \pm 0.5 \mathrm{~J} / \mathrm{mol} \mathrm{K}$. The combined results in eq (29) gives $\mathrm{d} H_{\mathrm{sm}} / \mathrm{d} T_{\mathrm{m}}=84.5 \pm 1.2 \mathrm{~J} / \mathrm{mol} \mathrm{K}$.

Clusius and Bartholome [1] and Kerr et al. [43] measured the specific heat of the solid along the vapor pressure curve. These data extrapolate to the triple point to yield $C_{P_{\text {ssm }}}=11.0 \pm 0.1 \mathrm{~J} / \mathrm{mole} \mathrm{K}$. This value combined with $\mathrm{d} H_{\mathrm{sm}} / \mathrm{d} T_{\mathrm{m}}, V_{\mathrm{sm}}$ from eq (25), and $\mathrm{d} P_{\mathrm{m}} / \mathrm{d} T_{\mathrm{m}}$ at the triple point give $\alpha_{\mathrm{sm}}=3.8 \pm 0.6 \times 10^{-3} \mathrm{~K}^{-1}$. This indirect estimate of the solid thermal expansivity lies about midway between the value derived from the work of Driessen et al. [14] and our direct result.

We began this section with a brief discussion of the problems that we met in applying our technique to solid samples. It is appropriate to conclude with some suggestions for improving the method. As we mentioned earlier, the major problem derives from the design of our pressure cell. Impressed changes in the sample volume always introduce radial inhomogeneities in the density. For solid deuterium, the very thin disk-shaped geometry of the sample combines with an inherently large internal friction. Together, they allow substantial pressure gradients to persist for prohibitively long periods of time.

We were unable to improve our results by annealing, even when this was done at pressures and temperatures very close to melting. We feel the only possible solution is to redesign the cell in a way that minimizes the radial density gradients. For example, it may be possible to construct a cell with a sample chamber thickness that is very nearly proportional to the displacement of the diaphragms. If this can be done, it is clear that changes in the sample volume will induce density gradients in the axial rather than the radial direction. Sample distortions can never be totally eliminated, but they should be far less severe in a carefully designed, lens-shaped sample than they were shown to be with our geometry. 


\subsection{Further Observations}

Finally, we discuss the effects of para to ortho conversion and their possible influence on the PVT results. We ran a number of experiments to measure such effects explicitly in both hydrogen and deuterium. For both isotopes, spin compositions were determined from the thermal conductivity of the gas samples [68]. On solidliquid mixtures of $\mathrm{n}-\mathrm{H}_{2}$, the measured time rate of change in $x_{\mathrm{p}}$, the para mole fraction, was $\mathrm{d} x_{\mathrm{p}} / \mathrm{d} t \simeq 0.01$ $\mathrm{h}^{-t}$. This result agrees with the self-conversion rate given by Woolley et al. [4]. Measurements of the melting pressure $P_{\mathrm{m}}$ and the liquid molar volume $V_{1}$ (close to the melting curve) as functions of time gave $\mathrm{d} P_{\mathrm{m}} / \mathrm{d} t \simeq 0.06$ bar $\mathrm{h}^{-1}$ and $\mathrm{d} V_{1} / V_{1} \mathrm{~d} t \simeq 7 \times 10^{-5} \mathrm{~h}^{-1}$. These results divided by $\mathrm{d} x_{\mathrm{p}} / \mathrm{d} t$ gives $\mathrm{d} P_{\mathrm{m}} / \mathrm{d} x_{\mathrm{p}} \simeq 6$ bar and $\mathrm{d} V_{1} / V_{1} \mathrm{~d} x_{\mathrm{p}} \simeq 7 \times 10^{-3}$, which are consistent with previous results: $P_{m}\left(\mathrm{p}-\mathrm{H}_{2}\right)-P_{\mathrm{m}}\left(\mathrm{n}-\mathrm{H}_{2}\right)$ from the data of Woolley et al. [4], Mills and Grilly [28], and Goodwin and Roder [31]; $V_{1}\left(\mathrm{p}-\mathrm{H}_{2}\right)-V_{1}\left(\mathrm{n}-\mathrm{H}_{2}\right)$ by Scott and Brickwedde [38] and Wallace and Meyer [69].

For liquid $\mathrm{n}-\mathrm{D}_{2}$, the rate of self conversion, $\mathrm{d} x_{\mathrm{o}} / \mathrm{d} t$, was estimated by Woolley et al. [4] to be $5 \times 10^{-5} \mathrm{~h}^{-1}$. For the solid, Motizuki [70] calculated the value $5 \times 10^{-4}$ $\mathrm{h}^{-1}$ which is in the range 2 to $8 \times 10^{-4} \mathrm{~h}^{-1}$ that Grenier and White [35] obtained by direct measurement. We measured the conversion rate of $\mathrm{n}-\mathrm{D}_{2}$ several times in our cell. The results for $\mathrm{d} x_{0} / \mathrm{d} t$ were between 1 and $3 \times 10^{-4} \mathrm{~h}^{-1}$ for observation periods of 60 to $90 \mathrm{~h}$. The data are reasonably consistent with the earlier theoretical and experimental work. The conversion rates are small, and we feel that our PV'T measurements are not significantly influenced. The following numerical estimates should illustrate this point.

One effect of self-conversion is an increase in $P_{\mathrm{m}}$ at constant temperature. We noted in previous discussion that the melting pressures reported by Bereznyak and Sheinina [24] on $0-\mathrm{D}_{2}$ exceed ours on $\mathrm{n}-\mathrm{D}_{2}(33.3 \%$ ortho $)$ by about 2 bar. This difference scales to 6 bar for $\Delta x_{\mathrm{p}}=1$, which is similar to the difference in $P_{\mathrm{m}}$ between pure ortho and para hydrogen. For the self-conversion rate $\mathrm{d} x_{\mathrm{o}} / \mathrm{d} t=2 \times 10^{-4} \mathrm{~h}^{-1}$, we should observe $\mathrm{d} P_{\mathrm{m}} / \mathrm{d} t \simeq 1.2 \times 10^{-3} \mathrm{bar} / \mathrm{h}$.

Another effect of conversion is an increase in the molar volume of the sample. An estimate of the fractional change in the liquid volume can be calculated from the equation

$$
\frac{\Delta V_{1}}{V_{1}}=-\beta_{1}\left(\frac{\partial P_{\mathrm{Q}}}{\partial x_{\mathrm{p}}}\right)_{T, V} \Delta x_{\mathrm{p}}
$$

where $\beta_{1}$ is the compressibility of the liquid, $x_{\mathrm{p}}$ is the fraction of the para spin modification in the mixture, and $P_{\mathrm{Q}}$ is an effective pressure introduced by the electric quadrupole interactions of the para species. We estimate the value $\left(\partial P_{\mathrm{Q}} / \partial x_{\mathrm{p}}\right)_{T, V} \simeq 12.3$ bar from table 1 of Driessen et al. [14]. For liquid compressibilities on the order of $7 \times 10^{-4}$ bar $^{-1}$, we calculate $\Delta V_{1} / V_{1} \simeq 8 \times 10^{-3}$ for $\Delta x_{\mathrm{p}}=1$, which is also similar to $H_{2}$. Thus in $n-D_{2}$ with $\mathrm{d} x_{\mathrm{p}} / \mathrm{d} t=-d x_{\mathrm{o}} / \mathrm{d} p, \quad$ we expect the rate $\mathrm{d} V_{1} / V_{1} \mathrm{~d} t \simeq 2 \times 10^{-6} \mathrm{~h}^{-1}$.

The longest times taken for the various measurements were $1 \mathrm{~h}$ for the liquid $\alpha$ and $\beta, 10 \mathrm{~h}$ for solid $\alpha$ and $\beta$, and $20 \mathrm{~h}$ for $\Delta V_{\mathrm{m}}$. The longest time of low-temperature exposure before $P_{\mathrm{m}}$ and $V_{1}$ measurements were made was $12 \mathrm{~h}$. Thus, in all cases the effects of ortho-para conversion are within the quoted accuracy limits of the data. It is nonetheless interesting to compare the values of $P_{\mathrm{m}}$ and $V_{1}$ at the beginning and at the end of the long runs (60 to $90 \mathrm{~h}$ ) involving $\Delta V_{\mathrm{m}}$.

Again, with an assumed conversion rate of $2 \times 10^{-4}$ $\mathrm{h}^{-1}$, we expect a fractional volume change in the liquid to be roughly $1.6 \times 10^{-4}$ for an 80 -h period. Volume changes of this magnitude are easily measured in our apparatus. Indeed the observations include such results, but there were also some changes that are much smaller and, in two cases, some large volume decreases $(1.7$ to $\left.1.9 \times 10^{-4}\right)$. However, volume losses were also observed in the $\mathrm{p}-\mathrm{H}_{2}$ measurements, where no conversion occurs, and these can be explained by a loss of the sample through a leaky cell valve.

For the melting pressure this self-conversion rate should produce an increase of about 0.10 bar for $n-D_{2}$ during an $80-\mathrm{h}$ period. Actual measured values, shown in table 6 , vary from -0.39 to +0.38 bar. The large decreases in $P_{\mathrm{m}}$ are not easily explained. If they were to

Table 6. Observed changes in the melting pressures of samples that had been held at low temperatures for 50 - to 70 -h periods.

\begin{tabular}{lrr}
\hline$T(\mathrm{~K})$ & Seq. No. $^{1}$ & $\Delta P_{\mathrm{m}}(\mathrm{bar})$ \\
\hline 18.83 & 9 & 0.00 \\
19.00 & 5 & -0.39 \\
19.20 & 8 & -0.28 \\
19.20 & 11 & 0.10 \\
19.40 & 4 & 0.31 \\
19.60 & 6 & -0.01 \\
19.80 & 3 & -0.06 \\
19.80 & 12 & 0.19 \\
20.00 & 7 & -0.30 \\
20.20 & 1 & 0.24 \\
20.20 & 2 & -0.03 \\
20.40 & 10 & -0.33 \\
20.40 & 13 & 0.04 \\
20.40 & 14 & 0.38 \\
\hline
\end{tabular}

${ }^{1}$ Chronological order of the measurements. 
be ascribed to bath temperature decreases, the $\Delta T$ would have to be on the order of $10 \mathrm{mK}$, which is far greater than our expected precision of a millikelvin or two.

There is evidence in some cases that a high initial measurement of $P_{\mathrm{m}}$ could have been made before the sample was adequately cooled; however, in other cases this explanation is unreasonable. For example, after the first run at 19.2 Kwas completed, we saw that a large decrease $\left(\Delta P_{\mathrm{m}}=-0.28\right.$ bar) had occurred in the melting pressure. We replaced the original sample with a fresh charge of $n-D_{2}$ from the storage bottle. The melting pressure of the second sample was then measured, and the result agreed with that obtained from the first sample when it was originally admitted to the cell and pressurized to cause freezing. The result removes the possibility of some kind of slow cooling of the cell, but it introduces the possibility of inadequate time for the liquid to be cooled or changed in some way with time.

Despite the lack of evidence for two solid structures in $\mathrm{n}-\mathrm{D}_{2}$, we did consider this possibility in connection with our observed melting pressure changes. If the Gibbs-energy functions of two solid phases are approximately equal, it should be quite easy to pressurize a liquid sample to the metastable solid form. It can be shown in this case that the observed $P_{\mathrm{m}}$ will be higher than that corresponding to the stable phase. At constant temperature the solid may then revert to the equilibrium structure, and as it does, the observed melting pressure will decrease. This argument is seen to hold for temperatures both above and below the assumed solid-solidliquid triple point.

Although, as we 'mentioned, there is no direct evidence for more than a single solid structure in $n-D_{2}$, we did observe some curious behavior with $\mathrm{p}-\mathrm{H}_{2}$ in our cell. Recall that Manzhelii [53,54] reported volume decreases of $0.15 \%$ in solid $\mathrm{p}-\mathrm{H}_{2}$ with small increases of temperature. In $\mathrm{p}-\mathrm{H}_{2}$ and $\mathrm{n}-\mathrm{H}_{2}$, Bereznyak and Sheinina [24] observed kinks in the freezing curve near $14.9 \mathrm{~K}$. Similarly, in our PVT apparatus, we observed $\Delta V \approx-0.07 \%$ in solid $\mathrm{p}-\mathrm{H}_{2}$ with slight increases of temperature. We also found the melting pressure of $\mathrm{p}-\mathrm{H}_{2}$ to decrease with time at rates of about $0.005 \mathrm{bar} \mathrm{h}^{-1}$. Over time periods of $60 \mathrm{~h}, P_{\mathrm{m}}$ decreases of approximately 0.3 bar were observed, similar to our measurements on $n-D_{2}$.

If this similarity supports the possibility that two (or more) solid phases exist in $n-D_{2}$, there is contrary evidence as well. In one of the long runs at $20.4 \mathrm{~K}$, a melting pressure change of -0.33 bar was observed. After the run was completed but before the lowtemperature valve was opened, the sample was held in the liquid phase for over an hour to assure that all of the solid had melted. The liquid was then refrozen and the melting pressure was again measured. The resulting value was identical to that obtained from the melting process only an hour before. This experiment tends to eliminate the possibility of a solid structure change with time. At best, it could indicate that such a change is fast when an "old" liquid is used. At this time, the anomalous changes of the melting pressure are not well understood.

\section{Summary and Conclusions}

The purpose of this study has been to provide an accurate and precise determination of the equation of state of $n-D_{2}$ in the region of the phase diagram near the triple point. We began by presenting measurements of the melting line. These data range in temperature from the triple point to $20.4 \mathrm{~K}$. The melting pressures were fit to a quadratic function of temperature. The average deviation of the points from the smooth curve is about 0.1 bar, which is comparable to the precision of the measurements. The observed regularity of these data contrasts to the anomalous behavior reported in similar studies [24] on p- $\mathrm{H}_{2}, \mathrm{n}-\mathrm{H}_{2}$, and $\mathrm{o}-\mathrm{D}_{2}$. The results of the earlier work were interpreted as evidence for the existence of solid-solid-liquid triple points in these systems. While this may be true, there appears to be no firm indication of a similar phenomenon in $n-D_{2}$.

We combined our melting data with existing vaporpressure measurements to establish an accurate triplepoint temperature. The result was corrected for the HD impurity measured by mass-spectrometric analysis. The value $T_{\mathrm{tp}}=18.723 \pm 0.010 \mathrm{~K}$ was given as the triple-point temperature of pure-phase $n-D_{2}$. A detailed comparison of this result to existing data is given elsewhere [33].

Data for the isothermal compressibility and thermal expansivity of the liquid were collected in the temperature range 18.8 to $21.0 \mathrm{~K}$ and at pressures between 4 and 70 bar. We compared these with estimates of corresponding values derived from existing molar volume data $[2,5,6]$. The most accurate and precise of these earlier data $[5,6]$ gave results that agree with ours to within a few percent. We also presented measurements of the molar volume of the liquid, which were made at nine points along the melting line. The combined liquid data were then fitted to a single empirical equation of state. The purpose of doing this is twofold. First, a description of the data by a single function is sufficient demonstration of their internal consistency. Second, the equation of state can be used to extrapolate beyond the limited range of the data and also to predict certain quantities that were not measured directly. This allows us to make indirect data comparison that would not otherwise be possible. 
As an example of this, we began by interpolating our equation of state to compare the molar volume with existing measurements $[2,6]$ at pressure. We then extrapolated the equation to the vapor-pressure curve where a number of direct measurements of the liquid molar volume $[1,5]$ exist. The analysis was finally extended by incorporating some of the existing calorimetric data for the liquid $[35,43,45]$. This additional information allowed us to compare our results with existing sound velocity [46-48] and isochoric heat capacity measurements [3]. The overall conclusion of the analysis is that our measurements agree with the best available data for differential quantities, such as the compressibility, ${ }_{1}(\partial P / \partial T)_{V}$, and sound velocity. However, small but significant uncertainties remain in the scale of the liquid data. Our volume measurements agree with those of Friedman et al. [6], but extrapolations of eq (8) to the vapor curve yield values that are at least $0.10 \mathrm{~cm}^{3} / \mathrm{mole}$ lower than the measurements of Kerr [5].

Results for the volume change on melting were then given. These were followed by a brief review of the existing evidence for a solid-solid phase transition in hydrogen. The behavior of our volume-change measurements showed no irregularities that would indicate a similar behavior in $n-D_{2}$.

From the volume-change measurements and the slope of the melting curve we derived the corresponding heats of fusion and fitted them to a linear function of pressure. These results agree with existing measurements at low pressures $[35,43]$. Finally, we derived solid molar volumes along the melting curve. The corresponding solid densities were fit to a linear function of the melting temperature. This empirical function was extrapolated to both low and high temperatures to compare with existing data. We found that our extrapolations are consistent with values derived from the best existing solid-phase data $[14,64]$.

We attempted to measure compressibilities and thermal expansion coefficients in the solid. The results of these measurements were found to be somewhat inconsistent with our solid densities along the melting curve. We briefly discussed the compressibility and thermal expansion coefficients that can be derived from the tabulated equation of state that Driessen et al. [14] presented. These results appear to be more nearly consistent with the observed solid compression. Finally, we derived an indirect estimate of the solid thermal expansion from a variety of data that had been presented earlier in the discussion. This calculation gave a higher value than we obtained from our direct measurements but lower than that deduced from the extrapolated equation of state.

The paper concluded with our unexplained observations of the changes of the melting pressure of the confined sample with time. We noted that these changes were opposite from what might be expected of the effects of ortho-para conversion.

The authors wish to thank Dr. R. L. Mills for participating in valuable discussions on topics in this paper and R. Jay Fries of the Laser Fusion Target Fabrication Group.

\section{References}

[1] Clusius, K., and E. Bartholome, Z. Physik. Chem. [B] 30, 237 (1935).

[2] Bartholome, E. Z. Physik. Chem. [B] 33, 387 (1936).

[3] Bartholome, E., and A. Eucken, Z. Electrochem. 42, 547 (1936).

[4] Woolley, H. W.; R. B. Scott and F. G. Brickwedde, J. Res. Natl. Bur. Stand. (U.S.) 41, 379 (1948).

[5] Kerr, E. C. J. Am. Chem. Soc. 74, 824 (1952).

[6] Friedman, A. S.; M. Trzeciak and H. L. Johnston, J. Am. Chem. Soc. 76, 1552 (1954).

[7] Prydz, R. Natl. Bur. Stand. (U.S.) NBS Reports 9276 (1967).

[8] Prydz, R.; K. D. Timmerhaus and R. B. Stewart, Adv. Cryog. Eng. 13, 384 (1968).

[9] Mills, R. L.; D. H. Liebenberg and J. C. Bronson, J. Chem. Phys. 68, 2663 (1978).

[10] Liebenberg, D. H.; R. L. Mills and J. C. Bronson, Phys. Rev. B 18,4526 (1978).

[11] Stewert, J. W. J. Phys. Chem. Sol, 1, 146 (1956).

[12] Anderson, M. S., and C. A. Swenson, Phys. Rev. B 10, 5184 (1974).

[13] Silvera, I. F.; A. Driessen and J. A. de Waal, Phys. Lett. 68A, 207 (1978).

[14] Driessen, A.; J. A. de Waal and I. F. Silvera, J. Low Temp. Phys. 34, 255 (1979).

[15] Grilly, E. R. Phys. Rev. 149, 97 (1966).

[16] Grilly, E. R. J. Low Temp. Phys. 4, 615 (1971).

[17] Grilly, E. R. J. Low Temp. Phys. 11, 33 (1973).

[18] Goodwin, R. D.; D. E. Diller, H. M. Roder and L. A. Weber, J. Res. Natl. Bur. Stand. (U.S.) 67, 173 (1963).

[19] Rubin, T.; H. W. Altman and H. L. Johnston, J. Am. Chem. Soc. 76, 5289 (1954).

[20] Roder, H. M.; L. A. Weber and R. D. Goodwin, Natl. Bur. Stand. Monograph 94 (Aug. 1965).

[21] Souers, P. C.; C. K. Briggs, J. W. Pyper and R. T. Tsugawa, Lawrence Livermore National Laboratory, Livermore, CA 94550, UCRL-52226 (1977).

[22] The International Practical Temperature Scale of 1968. Metrologia 5, 35 (1969).

[23] Grilly, E. R. J. Am. Chem. Soc. 73, 843 (1951).

[24] Bereznyak, N. G., and A. A. Sheinina, Fiz. Nizk. Temp. 6, 1255 (1980) [English trans., Sov. J. Low Temp. Phys. 6, 608 (1980)].

[25] Keesom, W. H.; A. Bilj and H. van der Horst, Leiden Comm. 217a (1931).

[26] Chester, P. F., and J. S. Dugdale, Phys. Rev. 95, 278 (1954).

[27] Mills, R. L., and E. R. Grilly, Phys. Rev. 99, 480 (1955).

[28] Mills, R. L., and E. R. Grilly, Phys. Rev. 101, 1246 (1956).

[29] Goodwin, R. D. Cryogenics 2, 353 (1962). 
[30] Younglove, B. A. J. Chem. Phys. 48, 4181 (1968).

[31] Goodwin, R. D., and H. M. Roder, Cryogenics 3, 12 (1963)

[32] Bedford, R. E.; M. Durieux, R. Muijlwijk and C. R. Barber, Metrologia 5, 47 (1969).

[33] Schwalbe, L. A., and E. R. Grilly (to be published in the J. Phys. Chem. Ref. Data)

[34] Bereznyak, N. G.; I. V. Bogoyavlenskii, L. V. Karnatsevich and V. S. Kogan, Zh. Eksp. Teor. Fiz. 57, 1937 (1969) [Sov. Phys. JETP 30, 1048 (1970)].

[35] Grenier, G., and D. White, J. Chem. Phys. 40, 3015 (1964).

[36] Grilly, E. R. J. Am. Chem. Soc. 73, 5307 (1951).

[37] Rudenko, N. S., and V. P. Slyusar', Zhur. Fiz. Khim. 43, 781 (1969) [Russ. J. Phys. Chem. 43, 434 (1969)].

[38] Scott, R. B., and F. G. Brickwedde, J. Res. Natl. Bur. Stand. (U.S.) 19, 237 (1937), J. Chem. Phys. 5, 736 (1937).

[39] Roder, H. M.; D. E. Diller, L. A. Weber and R. D. Goodwin, Cryogenics 3, 16 (1963).

[40] Weber, L. A.; D. E. Diller, H. M. Roder and R. D. Goodwin, Cryogenics 2, 236 (1962).

[41] White, D.; A. S. Friedman and H. L. Johnston, J. Am. Chem. Soc. 72, 3927 (1950).

[42] Onnes, H. K., and C. A. Crommelin, Leiden Comm. 137a (1913).

[43] Kerr, E. C.; E. B. Rifkin, H. L. Johnston and J. T. Clarke, J. Am. Chem. Soc. 73, 282 (1951).

[44] Roder, H. M.; G. E. Childs, R. D. McCarty and P. E. Angerhofer, Natl. Bur. Stand. (U.S), Tech. Note 641 (Oct. 1973).

[45] Brouwer, J. P.; A. M. Vossepoel, C. J. N. Van den Meijdenberg and J. J. M. Beenakker, Physica 50, 125 (1970).

[46] Bezuglyi, P. A., and R. Kh. Minyafaev, Fiz. Tverd. Tela 9, 3622 (1967) [Soviet Phys. Solid State 9, 2854 (1968)].

[47] Wanner, R., and H. Meyer, J. Low Temp. Phys. 11, 715 (1973).

[48] Pashkov, V. V., and E. V. Konovodchenko, Fiz. Nizk. Temp. 4, 436 (1978) [Sov. J. Low Temp. Phys. 4, 212 (1978)].

[49] Roder, H. M. Cryogenics 13, 439 (1973).

[50] Cook, G. A.; R. F. Dwyer, O. E. Berwaldt and H. E. Nevins, J. Chem. Phys. 43, 1313 (1965).

[51] Udovidchenko, B. G., and V. G. Manzhelii, J. Low Temp. Phys. 3, 429 (1970).
[52] Wallace, B. A., and H. Meyer, J. Low Temp. Phys. 15, 297 (1974).

[53] Manzhelii, V. G.; B. G. Udovidchenko and V. B. Esel'son, Zh. Eksp. Teor. Fiz. Pis'ma 18, 30 (1973) [Sov. Phys.--JETP Lett. 18, 16 (1973)].

[54] Manzhelii, V. G.; B. G. Udovidchenko and V. B. Esel'son, Fiz Nizk. Temp. 1, 799 (1975) [Sov. J. Low Temp. Phys. 1, 384 (1975)].

[55] Mills, R. L. J. Low Temp. Phys. 31, 423 (1978).

[56] Krupskii, I. N.; Yu. E. Stetsenko and G. N. Shcherbakov, Zh. Eksp. Teor. Fiz. Pis'ma 23, 442 (1976) [Sov. Phys.--JETP Lett. 23, 400 (1976)].

[57] Bereznyak, N. G., and A. A. Sheinina, Fiz. Nizk. Temp. 7, 685 (1981) [Sov. J. Low Temp. Phys. 7, 335 (1981)].

[58] Dwyer, R. F.; G. A. Cook, B. M. Shields and D. H. Stellrecht, J. Chem. Phys. 42, 3809 (1965).

[59] Dwyer, R. F.; G. A. Cook, O. E. Berwaldt and H. E. Nevins, J. Chem. Phys. 43, 801 (1965).

[60] Schuch, A. F., and R. L. Mills, Phys. Rev. Lett. 16, 616 (1966).

[61] Bostanjoglo, O. and R. Kleinschmidt, J. Chem. Phys. 46, 2004 (1967).

[62] Mucker, K. F; S. Talhouk, P. M. Harris, D. White and R. A. Erickson, Phys. Rev. Lett. 15, 586 (1965).

[63] Mucker, K. F.; P. M. Harris, D. White and R. A. Erickson, J. Chem. Phys. 49, 1922 (1968)

[64] Nielsen, M. Phys. Rev. B7, 1626 (1973).

[65] Yarnell, J. L.; R. L. Mills and A. F. Schuch, Fiz. Nizk. Temp. 1, 760 (1975) [Sov. J. Low Temp. Phys. 1, 366 (1975)].

[66] Kogan, V. S.; Yu. Ya. Milenko and T. K. Grigorova, Physica 53, 125 (1971).

[67] Constable, J. H.; C. F. Clark and J. R. Gaines, J. Low Temp. Phys. 21, 599 (1975).

[68] Grilly, E. R. Rev. Sci. Instr. 24, 72 (1953).

[69] Wallace, B. A., and H. Meyer. Proceedings of the 13th International Conference on Low Temperature Physics, Boulder, CO, Vol. 2, p. 194 (1972).

[70] Motizuki, K. J. Phys. Soc. Japan 17, 1192 (1962).

[71] Bereznyak, N. G., and A. A. Sheinina, Fiz. Nizk. Temp. 3, 804 (1977) [Sov. J. Low Temp. 3, 6 (1977)]. 